\title{
El efecto de la tecnología en las exportaciones manufactureras mexicanas hacia Estados Unidos*
}

\author{
Ana Lilia Valderrama Santibáñez** y Omar Neme Castillo***
}

\section{RESUMEN}

El objetivo de este estudio es demostrar que en el largo plazo las exportaciones mexicanas hacia Estados Unidos son impulsadas principalmente por factores tecnológicos y de organización industrial. En particular, se identifica si las capacidades tecnológicas tienen significado sistemático, así como la función que desempeñan en el corto plazo. Para ello, se estima un modelo de determinantes de las exportaciones bajo diversas especificaciones diferenciadas por las variables tecnológicas. Se emplean técnicas de cointegración para una estimación rigurosa de las exportaciones en el largo plazo, incluyendo el VECM, que muestra la dinámica de corto plazo, y se determina si estas variables causan en el sentido de Granger a las exportaciones. Se observa que las capacidades tecnológicas y competencia monopólica impactan en las exportaciones.

Palabras clave: exportaciones, capacidades tecnológicas, cointegración.

Clasificación JEL: F12, F14, C22, O31, 033.

\begin{abstract}
The objective of this study is to demonstrate that in the long run Mexican manufacturing exports sent to the United States are mainly boosted by technological and industrial organization factors. Particularly it is identified whether technological capacities have a systematic significance as well as their role in the short run. To this end, a model of export determinants is estimated under several specifications differentiated by the technological variables. Cointegration techniques are employed to achieve a rigorous estimation of the long run exports, including the VECM, which shows the short run dynamic, and it is determined whether these variables Granger-cause the exports. It is observed that technological capacities and monopolistic competition affect exports.
\end{abstract}

Key words: exports, technological capacities, cointegration.

Clasification JEL: F12, F14, C22, O31, 033

* Fecha de recepción: 17 de junio de 2010. Fecha de aceptación: 29 de noviembre de 2010.

** Profesora de la Sección de Estudios de Posgrado e Investigación, Escuela Superior de Economía del IPN, México. Correo electrónico: analilia.vs@gmail.com.

*** Profesor de la Sección de Estudios de Posgrado e Investigación-Escuela Superior de Economía del IPN y de la Facultad de Ciencias Políticas y Sociales de la UNAM, México. Correo electrónico: onemeco@gmail.com. 


\section{INTRODUCCIÓN}

Las exportaciones manufactureras mexicanas registraron un avance considerable a mediados de los años noventa, motivadas por un aumento en las elasticidades ingreso en el comercio internacional (en bienes tecnológicos), del proceso globalizador, del mayor uso de las nuevas tecnologías y, en particular, de la integración comercial con Estados Unidos (EUA) mediante el acceso preferente vía TLCAN, que permitió elevar considerablemente la participación como fuente de importaciones de EUA, colocando a México como uno de los tres mayores exportadores a ese mercado. ${ }^{1}$ Asimismo, el dinamismo importador de EUA, que de 1990 a 2006 mostró un crecimiento promedio anual de 15\%, principalmente en productos tecnológicos, generó mayor espacio para las manufacturas mexicanas en ese mercado.

Al mismo tiempo, la evolución de ciertas variables en igual sentido que las exportaciones sugiere la existencia de una correlación entre éstas. Por ejemplo, la capacidad de producción (índice del volumen físico de la producción) creció en el periodo $100 \%$; el gasto en investigación y desarrollo (ID) de empresas privadas aumentó $560 \%$; el gasto total manufacturero en esas actividades se multiplicó por nueve; las patentes concedidas crecieron $770 \%$ y la participación de científicos e ingenieros en el trabajo manufacturero se duplicó.

En contraste, las variables vinculadas con el precio de las exportaciones mostraron un desempeño relativamente pobre; por ejemplo, los costos laborales crecieron de 1985 a 2006 en $130 \%$, como consecuencia del pobre aumento en productividad laboral de únicamente $40 \%$ y de un incremento de $140 \%$ de la tasa de salario; el índice del valor unitario de exportación redujo una cuarta parte de su valor, indicando el restringido desempeño en términos de calidad; el tipo de cambio real se apreció en promedio 30\% limitando la competitividad precio de las exportaciones, a pesar de la reducción del costo del uso del capital para estas actividades 60 por ciento.

En consecuencia, con el mejor desempeño de los factores "tecnológicos" durante el periodo frente a los factores "relacionados con el precio" en un contexto de alto dinamismo exportador de las manufacturas mexicanas, se argu-

\footnotetext{
${ }^{1}$ Este mecanismo de comercio fomentó el crecimiento y concentración de las exportaciones mexicanas en ese país, para representar $5.7 \%$ del total exportado por México en 1985 y $85 \%$ en 2006. De igual manera, permitió que las exportaciones pasaran de 26 mil millones de dólares en $1985,1.7 \%$ de las exportaciones totales del mundo, a 250 mil millones de dólares en 2006 (2.1\% de las exportaciones globales).
} 
menta que la competitividad de las exportaciones mexicanas en el mercado estadounidense está influenciada en mayor medida por variables tecnológicas. Al respecto, la bibliografía empírica encuentra que más que la competitividad precio de las exportaciones (precios relativos), la competitividad tecnológica (procesos tecnológicos y estructura de mercado) es fundamental para el desempeño exportador (Fagerberg, 1996). Krugman (1983) y Grossman y Helpman (1995) argumentan que la tecnología y diferenciación de producto influyen en el comercio internacional. Según éstos, algunos países exportan más porque mediante procesos tecnológicos endógenos (learning-by-doing e ID) y estructuras de mercado no competitivas aumentan las oportunidades y capacidades de exportación de las empresas.

Los estudios empíricos de la relación entre cambio tecnológico y comercio internacional se dividen en estudios a nivel macroeconómico (Soete, 1987; Van Hulst et al., 1991; Cotsomitis et al., 1991; Verspagen y Wakelin, 1997) y a nivel micro (Greenhalgh, 1990; Lefebvre et al., 1998; Bernard y Bradford, 2004; Roper y Love, 2002; Love y Mansury, 2009; Bleany y Wakelin, 2002). Las conclusiones en cualquier nivel de análisis son similares al encontrar vínculos positivos entre la actualización tecnológica (empleando diversas variables de tecnología: patentes, actividades de ID, etcétera) y el desempeño exportador (aproximado por diferentes indicadores: participación de mercado, probabilidad o decisión de exportar, volumen o valor de las exportaciones).

Particularmente, se consideran los procesos de innovación tecnológica como una alternativa mediante la cual los países pueden enfrentar la competencia del comercio internacional. En términos generales, señalan que las economías que realizan actividades persistentes de innovación superan a aquellas sin procesos innovadores o escasos.

Para el caso de México, la bibliografía de los determinantes tecnológicos de las exportaciones manufactureras es limitada. No obstante, existen documentos específicos, como el de Narula y Wakelin (1998), que en un estudio sobre 40 países evalúan la importancia de los determinantes a nivel agregado de la competitividad, definida como participación de mercado. Siguiendo un enfoque neoschumpeteriano, éste determina que la tecnología desempeña una función central, principalmente en las economías en desarrollo, entre ellas la mexicana. Asimismo, Montobbio y Rampa (2005) evalúan el impacto del cambio tecnológico y estructural en el desempeño exportador -exportaciones sectoriales y participación de mercado- en nueve países en desarrollo. En particular, determinan si la especialización tecnológica, el cambio estructural, nacional e internacional, 
inversión extranjera directa e ID afectan las cuotas de mercado de las exportaciones. En general, los estudios del tema encuentran resultados semejantes a los establecidos para países industrializados, confirmándose el vínculo positivo entre tecnología -innovación- $\mathrm{y}$ exportaciones.

De esta forma, con datos de la OCDE, este documento determina la influencia que tienen factores relacionados con el precio y con la tecnología sobre las exportaciones manufactureras mexicanas a Estados Unidos (EUA), desde un enfoque comparativo. Se establece como hipótesis que las capacidades tecnológicas, además de la estructura de mercado, impacta tanto en el largo como en el corto plazos en mayor medida que las variables relacionadas con el precio.

Para ello, se plantea un modelo general basado en la ecuación tradicional de exportaciones pero que incorpora los factores arriba señalados y asimismo emplea diversos indicadores indirectos (proxies) de tecnología. Estimar este modelo diferenciado por variables tecnológicas permite identificar si éstas determinan a las exportaciones en un horizonte de mayor plazo así como su contribución en el corto plazo, al tiempo que revela su significación sistemática a partir de esas especificaciones diferentes. Esto es, se busca conocer si la tecnología, en cualquiera de las mediciones consideradas -en contraste con las variables-precio-, afecta el equilibrio de las exportaciones manufactureras mexicanas en un contexto de cointegración multivariada. Al respecto, se emplean técnicas de cointegración para establecer si existen relaciones de equilibrio estables en el largo plazo. El uso de las técnicas de cointegración permite una evaluación rigurosa de las exportaciones en el largo plazo. Asimismo, se estima un modelo del vector de corrección de errores para establecer la dinámica de las exportaciones en el corto plazo, aplicándose pruebas de exogeneidad débil y de exclusión. Finalmente, se prueba si las variables explicativas determinan en el sentido de Granger a las exportaciones.

El resto del documento se organiza como sigue. La sección I presenta aspectos teóricos y empíricos de la función de exportaciones y de la relación entre innovación y exportaciones, así como las variables proxy de las capacidades tecnológicas. En la sección II se especifica el modelo a estimar. La sección III despliega los resultados. Por último se presentan las conclusiones.

\section{DETERMINANTES DE LAS EXPORTACIONES}

Esta sección comprende dos temas. Primero, se expone la función de exportación característica, punto de partida para la introducción de otras variables como 
determinantes potenciales de las exportaciones. Segundo, expone aspectos referentes a la relación entre la variable de innovación tecnológica y exportaciones.

\section{Modelo de determinantes de las exportaciones ampliado}

En la bibliografía predominan dos modelos generales: sustitutos perfectos (MSP) y sustitutos imperfectos (MSI); el primero supone que existe perfecta sustituibilidad entre los bienes homogéneos para consumo local y los de exportación, para los que existe un único precio mundial. El exceso de oferta doméstica para un nivel de precio determina las exportaciones.

No obstante, la mayoría de los estudios empíricos del comercio internacional de un país se han realizado a partir del MSI, en el que los bienes difieren de acuerdo con su país de origen. En éste, el bien exportado por el productor $i$ en el país $A$ es diferente del bien producido por el productor $j$ en el país $B$. En consecuencia, el precio de intercambio entre los países no es necesariamente único y la cantidad vendida por un país puede no depender del diferencial de precios entre las fuentes oferentes. Este modelo es más cercano a la realidad al considerar la diferenciación de producto y suponer que los precios y bienes internos y de exportación discrepan (Goldstein y Khan, 1986). Si los bienes internos y extranjeros son sustitutos perfectos y se producen bajo rendimientos constantes a escala, entonces todos los países se especializan y el nivel de comercio intraindustrial es bajo. Para el caso mexicano, se tiene que el peso del comercio intraindustrial en el total del comercio bilateral con EUA es mayoritario, lo que induce a pensar que el intercambio es entre bienes heterogéneos y, por ende, a preferir el MSI.

El MSI se clasifica en dos grupos; el primero representa al comercio mundial en un modelo simple de ecuaciones múltiples, donde cada ecuación individual estima las exportaciones de un país particular a todas las demás economías. El segundo examina los determinantes de las exportaciones entre economías. Este es el tipo de modelo que se considera en el presente trabajo; ${ }^{2}$ y en particular, el modelo de determinantes de exportaciones (MDDE), que es una combinación de los modelos de demanda y oferta de exportaciones en una ecuación reducida (Goldar, 1989). Específicamente, postula que los precios son fijos en el corto plazo y que no responden a fluctuaciones aleatorias en las exportaciones.

\footnotetext{
${ }^{2}$ El MSI se divide en cuatro modelos: $i$ ) modelos de demanda de exportaciones; $i$ ) modelos de determinantes de exportaciones; iii) modelos de ecuaciones simultáneas, y iv) modelos de dos regímenes. Para una revisión de los diferentes modelos de sustitutos imperfectos véase Goldstein y Khan (1986).
} 
La estructura básica de estos modelos se considera ad hoc con los factores tradicionales de competitividad de precios y con los factores que reflejen la actividad extranjera, así como con variables relativas a la presión de la demanda interna que representan el lado de la oferta. La presión de la demanda interna muestra teóricamente una relación negativa con las exportaciones. Un cambio positivo en la demanda interna lleva, dada una oferta, a que el sector exportador desvíe parte de su oferta hacia el creciente mercado nacional.

Así, en este trabajo se considera el MSI, con el que se supone que las exportaciones no son sustitutos perfectos de los bienes internos. En este sentido, la mayoría de trabajos empíricos de los determinantes de las exportaciones coinciden en que tanto factores de demanda como de oferta influyen en las exportaciones, siguiéndose la hipótesis de que éstas son un sistema de ecuaciones de oferta y demanda; lo que se puede expresar como:

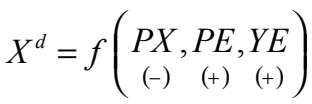

$$
\begin{aligned}
& X^{s}=g(\underset{(+)}{P X, P D,} \underset{(-)}{P(+-)})
\end{aligned}
$$

con $X^{d}$ como la demanda real de exportaciones, $X^{s}$ la oferta real de exportaciones, $P X$ el precio de los bienes de exportación, $P D$ el precio interno de esos mismos bienes; $Y D$ es una medida de capacidad de ingreso en el país exportador, $P E$ el precio extranjero de los bienes exportados desde la economía nacional, y $Y E$ es una variable de escala extranjera. Así, basados en el supuesto de la existencia de una relación lineal logarítmica entre las variables las ecuaciones (1) y (2) pueden reescribirse como:

$$
\begin{aligned}
& \ln X^{d}{ }_{t}=\gamma_{0}+\gamma_{1} \ln P X_{t}+\gamma_{2} \ln P E_{t}+\gamma_{3} \ln Y E_{t} \\
& \ln X_{t}^{s}=\alpha_{0}+\alpha_{1} \ln P X_{t}+\alpha_{2} \ln P D_{t}+\alpha_{3} \ln Y D_{t}
\end{aligned}
$$

La ecuación (4) de oferta de exportaciones se amplía para considerar el efecto de aspectos relacionados con la estructura de mercado y las capacidades tecnológicas. En cuanto al primero, las estructuras de mercado no competitivas, como la competencia monopolística, son una característica del comercio internacional actual, aceptándose que este tipo de organización industrial lleva a la dife- 
renciación de producto. De acuerdo con Krugman (1980), las exportaciones aumentan como consecuencia de la diferenciación de producto. Así pues, se espera una relación positiva entre poder de mercado y exportaciones.

Aparte, en los últimos años la innovación tecnológica reflejada en productos o procesos ha llegado a ser una de las variables más importantes en el análisis de las exportaciones. Si bien la evidencia empírica no es totalmente concluyente en este sentido, parece que en general las actividades tecnológicas influyen positivamente en las exportaciones (Madsen, 2004). La inversión en capacidades tecnológicas es decisiva para la acumulación de tecnología que sirve de base para una exportación exitosa (Bell y Pavitt, 1993). En consecuencia, la ecuación (4) puede expresarse como:

$$
\ln X_{t}^{s}=\alpha_{0}+\alpha_{1} \ln P X_{t}+\alpha_{2} \ln P D_{t}+\alpha_{3} \operatorname{lnYD_{t}}+\alpha_{4} \ln E M_{t}+\alpha_{5} \ln C T_{t}
$$

donde $E M_{t}$ es la estructura de mercado y $C T_{t}$ las capacidades tecnológicas, ambas en el tiempo $t$. El sistema de ecuaciones formado por (3) y (5) puede unirse en una ecuación que representa las exportaciones de equilibrio en el largo plazo. Para ello, las ecuaciones (3) y (5) se expresan primero en términos de $P X_{t}$ y después se igualan considerando que en el equilibrio de largo plazo la oferta $X^{s}$ y la demanda $X^{d}$ son iguales. En consecuencia, el modelo de los determinantes de las exportaciones es:

$$
\begin{aligned}
\ln X_{t}=\beta_{0} & +\beta_{1} \ln P D_{t}+\beta_{2} \ln P E_{t}+\beta_{3} \ln Y D_{t}+\beta_{4} \ln Y E_{t} \\
& +\beta_{5} \ln E M_{t}+\beta_{6} \ln C T_{t}+\varepsilon_{t}
\end{aligned}
$$

Con $\beta_{1}<0, \beta_{2}>0, \beta_{3}>0, \beta_{4}>0, \beta_{5}>0$ y $\beta_{6}>0$, y $\beta_{0}=\left[\gamma_{1} \alpha_{0}-\alpha_{1} \gamma_{0}\right] /\left[\gamma_{1}-\alpha_{l}\right]$,

$$
\begin{aligned}
& \beta_{1}=\left[-\alpha_{2} \gamma_{1}\right] /\left[\gamma_{1}-\alpha_{1}\right], \beta_{2}=\left[-\alpha_{3} \gamma_{1}\right] /\left[\gamma_{1}-\alpha_{1}\right], \beta_{3}=\left[\alpha_{1} \gamma_{2}\right] /\left[\gamma_{1}-\alpha_{1}\right], \\
& \beta_{4}=\left[\alpha_{1} \gamma_{3}\right] /\left[\gamma_{1}-\alpha_{1}\right], \beta_{5}=\left[\alpha_{1} \gamma_{4}\right] /\left[\gamma_{1}-\alpha_{1}\right] \text { y } \beta_{6}=\left[\alpha_{1} \gamma_{5}\right] /\left[\gamma_{1}-\alpha_{1}\right] .
\end{aligned}
$$

En este modelo, los bienes destinados al mercado de exportación y al nacional están diferenciados y la decisión de producir bienes para exportar o para el mercado local depende de los precios relativos. Emplear este modelo en el sector manufacturero parece razonable puesto que en esas industrias los bienes son altamente diferenciados. Por tanto, la función a especificar en este trabajo se considera como una forma reducida de un modelo de oferta-demanda con una sola ecuación que incorpora elementos de ambas partes. 


\section{Capacidades tecnológicas y exportaciones}

La bibliografía teórica señala la relación positiva entre procesos de innovación y desempeño exportador, particularmente, en términos de valor y participación de mercado. En este sentido, la naturaleza cuasi pública del conocimiento genera externalidades positivas a partir de spillovers y otros mecanismos como publicaciones o ingeniería de reversa, lo que permite el surgimiento de rendimientos crecientes en las industrias. En esencia, los costos de generación de conocimiento se pagan sólo una vez por lo que su difusión es virtualmente libre y sin costo. Por ende, el conocimiento puede adquirirse por esfuerzos propios y por el desbordamiento mundial del conocimiento. Así, el conocimiento es un bien no rival y puede emplearse simultáneamente por diferentes agentes sin costo adicional.

De esta manera, aunque los países y empresas no generen investigación y desarrollo (ID) y, en última instancia, nuevo conocimiento, pueden beneficiarse de él. Esta propiedad del conocimiento permite a países y empresas con menores capacidades tecnológicas acumular conocimiento más rápido mediante la adopción internacional de conocimiento y así reducir la brecha con las economías avanzadas. Al respecto, la bibliografía del catching up o "actualización tecnológica” identifica una relación positiva entre indicadores de innovación y exportaciones nacionales (Fagerberg y Verspagen, 2002).

Asimismo, los recursos tecnológicos pueden generar una doble ventaja competitiva para las economías y sus empresas. Por un lado, proporcionan ventajas en costos, al desarrollar nuevos y más eficientes procesos productivos y, por el otro, ventajas competitivas basadas en la diferenciación de producto, a partir de innovaciones de productos que permiten ofrecer éstos de acuerdo con las exigencias de los clientes o de una calidad superior.

Por último, la innovación afecta las exportaciones por dos vías. Primero, las innovaciones tienen efecto transitorio en las ganancias de las empresas al alterar su posición competitiva en el corto plazo. Cuando una empresa introduce una innovación obtiene poder de mercado temporal, el cual genera mayores beneficios hasta que otras empresas imiten esa innovación. Segundo, las innovaciones generan una diferencia estructural entre empresas innovadoras y no innovadoras, ya sea porque desarrollan diferentes capacidades o porque la innovación requiere de costos hundidos, que no todas las empresas pueden cubrir. Por ende, la innovación afecta positivamente a las exportaciones.

Aparte, la bibliografía del tema utiliza un amplio conjunto de variables independientes para modelar la relación entre exportaciones e innovación, aun- 
que no refleja un consenso acerca de la proxy de innovación que debe utilizarse. En este sentido, los indicadores de las actividades de innovación utilizados en países desarrollados (tales como el número de patentes y gastos en ID) no son del todo satisfactorios en el caso de países en desarrollo (Lall, 2000). En consecuencia, en este documento se considera un conjunto de indicadores relacionados con tres aspectos del proceso de innovación: $a$ ) esfuerzo innovador; $b$ ) adquisición de tecnologías, y $c$ ) resultados innovadores.

\section{a) Esfuerzo innovador}

Las actividades en ID se emplean para representar las capacidades tecnológicas; las proxies utilizadas son el gasto en ID en el total del sector manufacturero como proporción del PIB agregado (iid1), el valor de los gastos en ID de empresas privadas manufactureras en miles de millones de dólares de 2000 (iid2) y el acervo de gastos en ID de iid2 (iid3). Las series se toman de la Stan Data Base de la OCDE y se expresan en términos constantes $(2000=100)$ mediante el índice de precios implícito del PIB.

Una de las contribuciones de este documento es que emplea tanto variables flujo como acervo para medir el impacto de la tecnología en las exportaciones. De acuerdo con Madsen (2004), la acumulación es la medida relevante porque refleja el potencial de exportación dado que el acervo tecnológico está constantemente depreciándose; por ende, las actividades de ID necesitan conservarse en cierto nivel para mantener ese acervo constante sin experimentar una caída en la producción y exportación. Para construir el acervo de capital tecnológico mediante el método usual del inventario permanente se requieren tres elementos: $i$ ) una medida del acervo inicial, ii) el deflactor de los gastos corrientes de ID (deflactor del PIB), y iii) la tasa de depreciación del acervo de ID; como no existe evidencia ni teórica ni empírica clara de esta tasa, se prueban tasas alternativas (del 5, 7, 10, 15 y 20\%) y se elige la que mejor explique el efecto del acervo tecnológico en las exportaciones.

El acervo de capital tecnológico en $t$ está dado por $s k t_{t}=(1-\delta) \cdot s k t_{t-1}+I_{t-1}$, donde $\delta$ es la tasa de depreciación e $I$ es la inversión realizada en el periodo anterior en actividades de ID. Para aproximar el acervo de capital tecnológico en $t-1$ $\left(s k t_{t-1}\right)$, se emplea la razón capital-producto $(k)$, con $k=[(1-\delta) \cdot k+i] /[1+g]$, donde $i$ es la razón inversión-producto (ID/PIB) y $g$ la tasa de crecimiento de la inversión. El producto se aproxima mediante el PIB y la inversión a través de la inversión en ID. La tasa de crecimiento se calcula usando el logaritmo de la tasa 
de crecimiento promedio anual en el periodo $1985-2006$, esto es, $\ln \left(\operatorname{Iy} D_{2006} /\right.$ $\left.I+D_{1985}\right) / 22$. La razón inversión-producto se calcula como el promedio de los 22 años incluidos. ${ }^{3}$ Los datos se obtienen de la Stan Data Base de la OCDE.

Adicionalmente, cabe esperar que la inversión en ID realizada en EUA tenga algún efecto en las exportaciones manufactureras mexicanas, principalmente porque algunas empresas de EUA realizan actividades de ID en su territorio y transfieren parte de ese nuevo conocimiento (innovación de producto o proceso) a sus filiales en México para la manufactura de bienes diferenciados que más tarde son exportados a su propio mercado, aprovechando así las ventajas temporales de poder de mercado generadas por la ID. Por tanto, el acervo de capital tecnológico en EUA (skteu) al que tienen acceso las empresas mexicanas parece influir positivamente en las exportaciones. Este indicador se construye de la misma forma que el anterior, pero considerando los datos para Estados Unidos.

Aparte, el capital humano tiende a reflejar una relación positiva con las exportaciones. El acervo de capital humano que trabaje en ID representa mayores oportunidades para generar patentes y contribuir al mejor uso de recursos de las empresas. Al respecto, las cuatro proxies de capital humano empleadas son función del grado de educación: $i$ ) número de personas con estudios técnicos, de licenciatura y de posgrado (OCDE, 2007), en miles de personas ( $h$ ); ii) participación del trabajo calificado en el total del trabajo manufacturero (Dunning, 1995), en porcentajes $(k h)$; iii) participación de científicos e ingenieros en el total del trabajo en la manufactura (Van Dijk, 2002), expresada en términos porcentuales ( $k h l$ ), y $i v$ ) total de egresados de posgrado como porcentaje del total del trabajo en la industria manufacturera (Narula y Wakelin, 1998) (kh2). Los datos se obtuvieron del Sistema Integrado de Información sobre Investigación Científica y Tecnológica del Conacyt y se combinaron con datos de manufacturas de la Stan Data Base de la OCDE.

\section{b) Adquisición de tecnologías}

La adquisición de tecnologías es determinante de las exportaciones mediante diversas variables, entre las que están la inversión en tecnologías incorporadas (compra de equipo y maquinaria nacional o extranjera), intensidad del capital, mejoras en los procesos de producción (automatización), y adquisición de tecno-

${ }^{3}$ Alternativamente se consideró a i como el nivel de inversión inicial, esto es, el gasto en ID en 1985, sin embargo, los resultados no presentan modificaciones importantes. 
logías no incorporadas (licencias o derechos por know how). La influencia positiva de la adquisición de tecnologías incorporadas en el nivel exportador se debe a los efectos de aprendizaje y escala relacionados con la nueva maquinaria y equipos (Van Dijk, 2002).

Dada la restricción de datos, se mide indirectamente la adquisición de tecnologías por medio de dos tipos de variables relacionadas con la innovación de proceso y de producto. Primero, las empresas en los países en desarrollo consideran las actividades en ID como una forma de adaptar productos existentes y mejorar su calidad, asimilando el conocimiento existente. Esta actividad se basa en ID y principalmente en la importación de maquinaria y equipo (Lall, 2000) que, una vez dentro de la economía interna, afecta la producción nacional y presumiblemente las exportaciones. Para determinar el efecto de la adquisición de tecnología en las exportaciones, se usa la formación bruta de capital fijo importada por el sector manufacturero en miles de millones de dólares de $2000(a t){ }^{4}$

Alternativamente, se acepta que las importaciones de bienes desde una economía tecnológicamente más avanzada implica la adquisición de la tecnología incorporada en esos bienes; así, se asume que las importaciones de bienes intermedios facilitan mejoras en los productos y, por tanto, se utiliza para medir indirectamente la innovación de producto. Asimismo, la tecnología incorporada en las compras foráneas de bienes de capital permite mejoras en los procesos productivos, por lo que mide indirectamente la innovación de proceso (Delgado et al., 2002). En consecuencia, se prueba la importancia de la innovación de proceso (iproc), medida por las importaciones de bienes de capital, y de la innovación de producto (iprod), mediante las importaciones de bienes intermedios. Los datos se extraen del Banco de Información Económica del INEGI y se llevan a precios constantes $(2000=100)$, empleando el índice de precios implícito del PIB manufacturero.

\section{c) Resultados innovadores}

Los indicadores relacionados con las patentes, aunque imperfectos, son las mejores medidas para estudiar el efecto de la tecnología sobre las exportaciones (Madsen, 2004), ya que son producto directo de las actividades de ID formales o informales. Este trabajo emplea patentes y otros indicadores derivados. La pri-

\footnotetext{
${ }^{4}$ Alternativamente se considera la formación bruta de capital fijo importada por el sector manufacturero rezagada un periodo ( $\operatorname{atr} 1)$, en miles de millones de dólares $(2000=100)$.
} 
mera proxy es el número de patentes concedidas en México (patcon) en el periodo $t$ y la acumulación de patentes (patcon1); una variable menos exacta es el número de patentes solicitadas (patsol), que se espera menos significativa. Alternativamente, se consideran las patentes por persona, ya que reflejan la intensidad de la actividad innovadora y, en consecuencia, el potencial de nuevos productos. Esta variable se incluye en el modelo como patper, definida como el número de patentes concedidas por graduados del tercer nivel, que son los que tienen mayores capacidades para la innovación. Asimismo, se emplea la razón innovadora (ri), que se define como el número de patentes por empleado.

Aparte, las patentes externas son potencialmente más importantes para las exportaciones que las obtenidas domésticamente, ya que brindan mayor protección en los mercados internacionales (Magnier y Toujas-Bernate, 1994). En consecuencia, los exportadores tienen incentivos para patentar sus inventos en los mercados de exportación. Por ello, se incluye el número de patentes solicitadas por empresas mexicanas en EUA (spmxeu). Las estadísticas provienen de la base Highlights on Industrial Property Statistics de la Organización Mundial de la Propiedad Intelectual (WIPO, por sus siglas en inglés) y de la base Main Science and Technology Indicators de la OCDE. Adicionalmente, siguiendo la propuesta de Helpman (1981), además del gasto en ID, se incluyen uno y dos rezagos como determinantes de las exportaciones, puesto que se espera que las actividades de ID se materialicen con un desfase (iidrl e iidr2, respectivamente).

Por último, para captar con mayor precisión la relevancia de los factores tecnológicos se construyen dos indicadores de competitividad multilateral que permite la competencia de terceros países exportadores y de productores en el mercado de EUA. El primero señala la importancia de las patentes concedidas en la economía doméstica respecto a las patentes de las que disfrutan las empresas en otros países, ponderado por el peso de cada país competidor en el comercio con EUA, se conoce como índice relativo de producción de patentes (ipr) y está definido por: $i p r=\prod_{i}\left[\left(\text { pat }_{j}\right) / \text { pat }_{i}\right]^{\wedge}\left[w_{i}\right]$, donde pat $_{j}$ es el número de patentes concedidas en el país $j$ (México) dentro de la industria manufacturera, pat $_{i}$ es el número de patentes concedidas en el país $i$ (EUA) y $w_{i}$ es la participación de las exportaciones del país $i$ en el total exportado al mercado de EUA.

El segundo indicador es el índice de competitividad tecnológica (ict), que relaciona las exportaciones mexicanas a EUA potenciadas por las patentes concedidas en México respecto a las exportaciones totales del mundo a EUA potenciadas por las patentes en el mundo (Madsen, 2007). Se define como 
$i c t_{i}=\sum_{j=1}^{n}\left[\left(p_{j i}\right)\left(x_{j i}\right)\right] /\left[\left(p e_{j}+p d_{j}\right)\left(x t_{i}\right)\right]$, donde $i c t_{i}$ es el índice de competitividad tecnológica del país $i$ (México), $p_{j i}$ el acervo de patentes en el país $j$ (EUA) de los exportadores en el país $i, x_{j i}$ las exportaciones del país $i$ al mercado $j, p e_{j}$ el acervo de patentes externas en el país $j, p d_{j}$ el acervo de patentes nacionales en el país $j$ y $x t_{i}$ las exportaciones totales del país $i$. Las series se generaron a partir de datos obtenidos de las fuentes señaladas arriba.

Así, al considerar los distintos indicadores de innovación se espera una fuerte relación positiva de largo y corto plazos con las exportaciones. En la siguiente sección se especifica el modelo general a estimar y se define el resto de las variables (no tecnológicas) incluidas.

\section{VARIABLES Y EL MODELO}

En este documento se estima un modelo econométrico general mediante diversas proxies de tecnología para considerar las diferentes elasticidades de las exportaciones y en particular, determinar cuáles variables tecnológicas afectan las exportaciones mexicanas hacia EUA. Para ello, se parte del modelo tradicional donde las exportaciones manufactureras agregadas son función de los precios, de la demanda extranjera y de la capacidad de producción interna incorporando la estructura de mercado y la competitividad tecnológica. ${ }^{5}$ De esta manera, las siguientes variables se incluyen en la función de exportaciones.

\section{1) Demanda extranjera}

Se espera que las exportaciones se relacionen directamente con la actividad económica en el exterior. En este trabajo se analizan los efectos sobre las exportaciones de la demanda extranjera aproximada por las importaciones totales esta-

\footnotetext{
${ }^{5}$ Se reconoce que la entrada de China a la OMC ha modificado la competencia en el mercado de EUA, afectando el desempeño exportador de las manufactureras mexicanas. Por ende, se acepta que las exportaciones dependen de otros factores, fuera del alcance de este estudio, como competitividad estructural, IED, distancia económica, tamaño relativo de mercado, costos de transporte, capacidades, organizaciones, etcétera. Asimismo, como el documento se centra en las manufacturas agregadas, esto es, no controla la heterogeneidad entre industrias, las diferentes elasticidades de exportación podrían estar sesgadas hacia arriba. Por tanto, una extensión de este estudio debería incluir alguna de estas variables de control, seguir un enfoque de mayor desagregación -nivel industria o planta- y emplear técnicas de datos de panel de tal manera que se encuentren explicaciones alternativas.
} 
dounidenses $(\mathrm{meu})$. El signo esperado con las exportaciones es positivo. Las series se obtuvieron de la Stan Data Base de la OCDE y se llevaron, mediante el índice implícito del PIB, a miles de millones de dólares constantes $(2000=100)$.

\section{2) Precios relativos}

En este trabajo se sigue la configuración de precios relativos, por lo que las exportaciones están determinadas por los precios vigentes en la economía nacional y por los precios en el mercado de destino. La variable proxy es el costo unitario relativo de la mano de obra. Siguiendo a Graf (1996), los costos unitarios de la mano de obra $(\mathrm{cul})$ en la manufactura son los sueldos y salarios pagados a los trabajadores divididos por el producto real. En este sentido, los costos unitarios relativos de la mano de obra ( $c u r l$ ) derivan del cul promedio de los socios comerciales de un país dado (en este caso de EUA que representa el precio promedio de los bienes de terceros países y nacionales) que se dividen por el cul de México convertido a dólares, esto es curl $_{t}=\left[\left(\mathrm{cul}^{e u}{ }_{t}\right) / \mathrm{cul}^{\text {mex }}{ }_{t}\right] \times e_{t},{ }^{6}$ donde $\mathrm{curl}_{t}$ es el costo unitario relativo laboral en el tiempo $t, \mathrm{cul}^{e u}{ }_{t}$ el costo unitario laboral de EUA, cul $^{\text {mex }}{ }_{t}$ el costo unitario laboral de México y $e_{t}$ el tipo de cambio nominal; curl $_{t}$ es una medida de competitividad-precio, por lo que al emplear precios relativos, la relación con las exportaciones es positiva. ${ }^{7}$

\section{3) Capacidad de producción}

De acuerdo con Goldstein y Khan (1986), entre más alta sea la capacidad de producción de una economía, mayor será su capacidad de oferta exportable. En este sentido, el argumento de ventas-por-superávit sugiere que, dado un nivel de capacidad de producción, los exportadores sólo venderán al exterior si no son capaces de colocar sus productos en el mercado nacional. Se sigue la propuesta de la UNCTAD (2004) que mide esta variable a partir del índice de volumen físico

\footnotetext{
${ }^{6}$ En realidad, el costo unitario relativo de la mano de obra se determina por la expresión curl $_{i t}=\left\{\left[\left(\sum_{j=1}^{n} c_{l}\right) / n\right] /\left(\right.\right.$ cul $\left.\left.^{\text {mex }}{ }_{i t}\right)\right\} \times\left(e_{i t}\right)$, donde el término entre corchetes es el costo unitario promedio de los $n$ socios comerciales de México.

${ }^{7}$ Se utilizaron tres series. La primera se obtuvo directamente de la Stan Data Base de la OCDE. La segunda se calculó mediante la ecuación de arriba con datos de cul generados por la OCDE. En la última se obtuvieron datos de sueldos (compensación por hora) y del PIB de la base International Labor Comparison de la Oficina de Estadística Laboral de EUA para construir el indicador curl. La que muestra los mejores resultados en la estimación del modelo es la que finalmente se incluye en la función de exportaciones.
} 
de la producción (ivfp) que se extrae del Banco de Información Económica del INEGI y se llevó al año base 2000. Se espera una relación directa entre exportaciones y capacidad de producción.

\section{4) Competencia monopolística}

De acuerdo con Helpman (1981), la competencia monopolística es característica del flujo exportador, al permitir participar en mercados internacionales con bienes diferenciados. En principio, la presencia de competencia monopolística puede llevar a mayores exportaciones si existen barreras al comercio y la empresa puede segmentar los mercados, discriminando en el mercado interior y en el exterior. No obstante, la profundización de la competencia monopolística puede limitar las economías de escala y, por ende, llevar a menores exportaciones. En este trabajo se asume que las estructuras de mercado no competitivas son base para la exportación.

En consecuencia, el efecto de una estructura de mercado no competitiva en las exportaciones se prueba usando el índice de Lerner (il). Bajo el supuesto de rendimientos constantes a escala, este índice está dado por $i l=\left(p_{i}-c_{i}\right) / p_{i}$, donde $p_{i}$ es el precio promedio en la industria $i$, empleándose como proxy el índice de precios implícito, y $c_{i}$ es el costo marginal promedio al que se enfrentan las empresas en esa industria; en la práctica se emplea el índice del valor unitario. Este índice se construye dividiendo el valor de las ventas internas por su volumen y después se lleva al año base 2000. Los datos se obtienen de la Encuesta industrial anual del INEGI (varias ediciones). Así, se genera un $i l$ por cada clase de actividad y después se agrega para el total manufacturero mediante el promedio ponderado de estos índices. Se espera que el $i l$ muestre signo positivo con las exportaciones. La variable dependiente exportaciones $(x)$, se mide en miles de millones de dólares de 2000, y se obtuvo de la Stan Data Base de la OCDE. En el cuadro 1 se presentan las principales estadísticas descriptivas de las series empleadas.

De esta manera, se establece un modelo econométrico general, derivado de la función de producción neoclásica estándar, representado por una función de exportaciones, donde se muestra la relación entre variables "explicativas", importaciones de EUA ( $\mathrm{meu}$ ), el precio relativo ( $\mathrm{curl}$ ), volumen físico de la producción manufacturera (ivfp), competencia monopolística (il), procesos de innovación ( $h$, iidl, etc.) y exportaciones $(x)$. Basados en el supuesto de una relación lineal logarítmica entre las variables el modelo se formula como: 


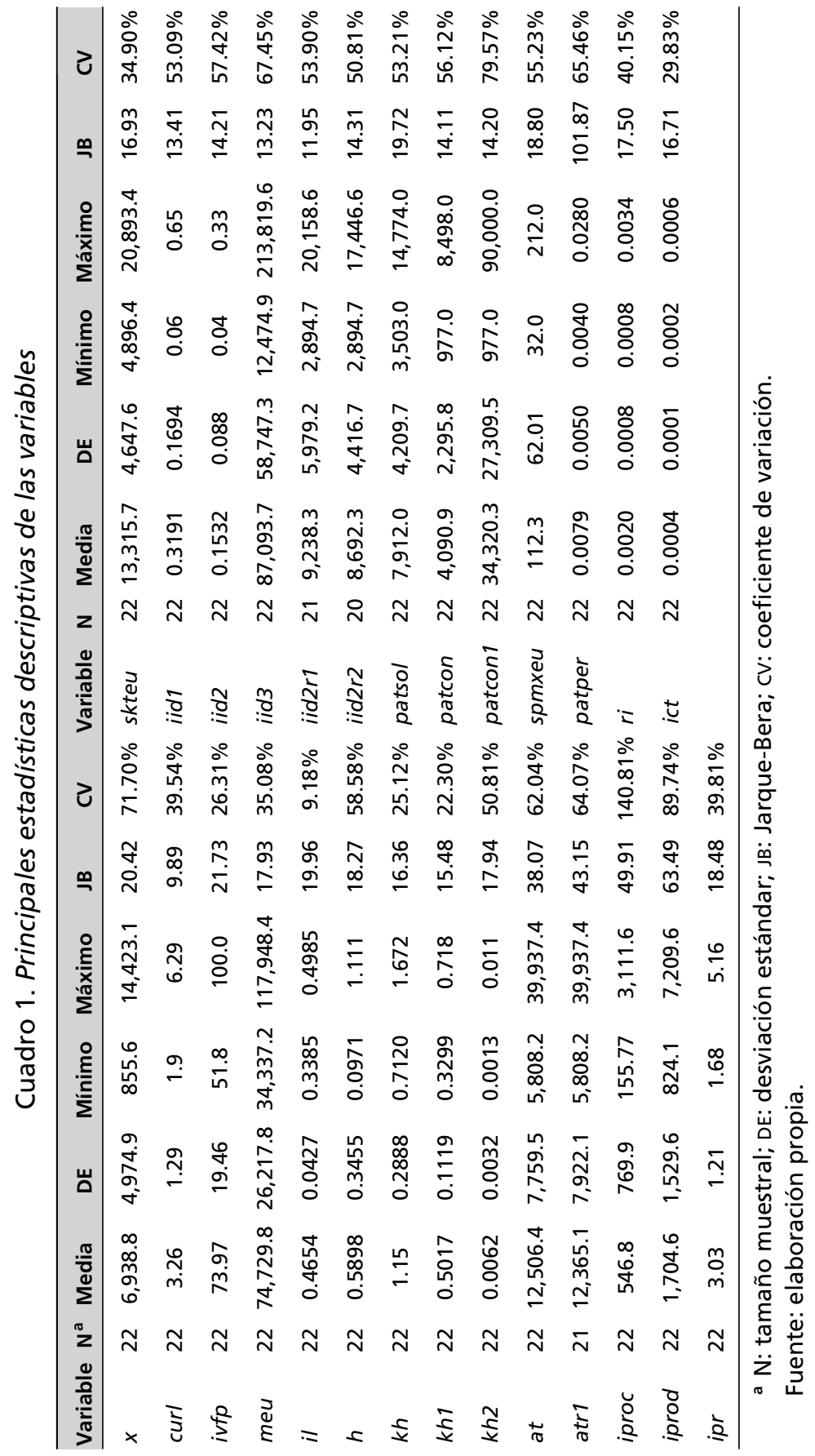




$$
\ln X_{t}=\beta_{1}+\beta_{2} \operatorname{lnpr} r_{t}+\beta_{3} \operatorname{lnde}+\beta_{4} \operatorname{lncp}_{t}+\beta_{5} \operatorname{lncm}_{t}+\beta_{6} \ln _{\text {innov }_{t}}+u_{t} .
$$

Esta ecuación es una forma reducida del modelo de oferta-demanda con una sola ecuación con elementos de ambas partes. La ecuación (7) se estima en la siguiente sección. Se sigue una metodología de cuatro etapas. Primero, se obtiene el orden de integración de las series temporales probando la existencia de una raíz unitaria. Segundo, si las series son integradas del mismo orden, se prueba la existencia de relaciones de cointegración entre las cinco variables de competitividad y las exportaciones a EUA. Tercero, se estima el vector de corrección de errores y, por último, se determina si las variables independientes causan en el sentido de Granger a las exportaciones.

\section{RESULTADOS}

El objetivo de este trabajo es determinar la función que desempeña cada una de las variables tecnológicas sobre las exportaciones. Por ende, se especifica un modelo general estimado con cinco variables explicativas, una de las cuales es el indicador de tecnología, que se calcula indirectamente por 22 diferentes proxies de innovación, para hallar la contribución sistemática de dicha variable en el largo plazo así como su dinámica de corto plazo. El rango del análisis comprende el periodo 1985-2006 con frecuencia anual. Las series se llevan a logaritmos naturales, ya que permiten disminuir la fluctuación de las variables y obtener series más homogéneas, eliminando así problemas de varianza. El modelo se estima considerando la inestabilidad de las exportaciones, por lo que cabe esperar que sea no estacionario y, por ende, se emplean técnicas de cointegración para su estimación.

La metodología de cointegración requiere primero determinar el orden de integración de las variables. El cuadro 2 muestra lo resultados de las pruebas ADF, PP y KPSS en niveles y en primera diferencia aplicadas a las series. Los resultados son variados; precios y demanda extranjera se comportan como procesos de caminata aleatoria. El il y $c p$ son integradas de orden cero, mostrando tendencia estacionaria. Las variables que representan el proceso de innovación tienden a comportarse como procesos ruido blanco, con algunas excepciones.

Por tanto, las variables se introducen en el modelo en niveles, ya que una combinación lineal de dichas variables será estacionaria. Esta especificación conserva la información intrínseca en las variables en niveles. Una forma de estimar las relaciones dinámicas entre las variables en un sistema, es especificar un 
modelo de vectores autorregresivos (VAR) y aplicarle técnicas de cointegración para verificar la existencia de relaciones de largo plazo. Primero, se establece el número de rezagos apropiados para la estimación del modelo VAR, tal que los residuos sean ruido blanco. El mejor modelo es el que minimiza el criterio de información de Akaike (AIC) y maximiza el estadístico LR. Los dos criterios indican que se debe incluir un rezago en el modelo VAR para la prueba de cointegración, por lo que la formulación más adecuada para el análisis de cointegración es $\operatorname{VAR}\left(k^{*}=1\right){ }^{8}$

Cuadro 2. Resultados de las pruebas de estacionariedad: ADF, PP y KPSS ${ }^{a}$

\begin{tabular}{|c|c|c|c|c|c|c|c|c|c|c|c|c|}
\hline \multirow{2}{*}{\multicolumn{2}{|c|}{ Variable N/D }} & \multicolumn{3}{|c|}{ Estadístico y decisión } & \multirow{2}{*}{\multicolumn{2}{|c|}{ Conclusión }} & \multirow[t]{2}{*}{ Variable } & \multirow[t]{2}{*}{ N/D } & \multicolumn{3}{|c|}{ Estadístico y decisión } & \multirow{2}{*}{$\begin{array}{l}\text { Conclu- } \\
\text { sión }\end{array}$} \\
\hline & & ADF & PP & KPSS & & & & & ADF & PP & KPSS & \\
\hline$x$ & D & $(-6.5067) *$ & $(-7.3411) *$ & $(0.1590)$ & & $\mathrm{I}(1)$ & iproc & $\mathrm{N}$ & $(-3.3137) * * *$ & * $(-6.6523) *$ & $(0.1560) * *$ & $\mathrm{I}(0)$ \\
\hline$i v f p$ & D & $(-3.2556) * * *$ & $(-3.3135) * * *$ & * $(0.1372)$ & *** & $\mathrm{I}(1)$ & iprod & $\mathrm{N}$ & $(-6.8455)^{*}$ & $(-3.8066) * *$ & $(0.1147) * * *$ & * $\mathrm{I}(0)$ \\
\hline curl & D & $(-3.2847) * * *$ & $(-3.3696) * * *$ & * $(0.0721)$ & *** & $I(1)$ & $h$ & D & $(-5.0676) *$ & $(-6.8347)^{*}$ & $(0.1821) * *$ & $\mathrm{I}(1)$ \\
\hline meu & D & $(-4.7631) *$ & $(-4.8229) *$ & $(0.0805)$ & *** & $\mathrm{I}(1)$ & $k h$ & D & $(-3.9543) * *$ & $(-3.9543) * *$ & $(0.1106) * * *$ & * $I(1)$ \\
\hline il & $\mathrm{N}$ & $(-3.8216) *$ & $(-4.4469) * *$ & $(0.1187)$ & *** & $\mathrm{I}(0)$ & kh1 & D & $(-4.0888) * *$ & $(-4.0888) * *$ & $(0.1106) * * *$ & * $I(1)$ \\
\hline at & $\mathrm{N}$ & $(-4.5118)^{*}$ & $(-4.4943)^{*}$ & $(0.1434)$ & *** & $\mathrm{I}(0)$ & kh2 & $\mathrm{N}$ & $(-3.9545) * *$ & $(-3.9545) * *$ & $(0.1226) * * *$ & * $I(0)$ \\
\hline atr1 & 2D & $(-4.0763) * *$ & $(-5.2418) *$ & $(0.3425)$ & & I(2) & spmxeu & D & $(-5.0016) *$ & $(-3.3099) * * *$ & $*(0.0705) * * *$ & * $I(1)$ \\
\hline iid 1 & $\mathrm{~N}$ & $(-5.6557) *$ & $(-5.4423) *$ & $(0.1953)$ & & $I(0)$ & skteu & D & $(-4.5834) *$ & $(-4.5832) *$ & $(0.0965) * * *$ & * $I(1)$ \\
\hline iid2 & $\mathrm{N}$ & $(-3.3673) * * *$ & $(-4.6007) *$ & $(0.1396)$ & *** & $I(0)$ & $r i$ & D & $(-5.1016) *$ & $(-7.0498) *$ & $(0.5000) *$ & $\mathrm{I}(1)$ \\
\hline$i i d 2 r 1$ & $\mathrm{~N}$ & $(-3.1584) * * *$ & $(-5.0438) *$ & $(0.1423)$ & *** & $I(0)$ & patcon & D & $(-5.9614)^{*}$ & $(-6.9157) *$ & $(0.5000) *$ & I(1) \\
\hline$i i d 2 r 2$ & $\mathrm{~N}$ & $(-3.0240) * * *$ & $(-4.8042) *$ & $(0.1428)$ & *** & $I(0)$ & patcon 1 & $\mathrm{~N}$ & $(-4.1106) * *$ & $(-13.8834)^{*}$ & $(0.1739)^{* *}$ & $\mathrm{I}(0)$ \\
\hline iid3 & $\mathrm{N}$ & $(-3.6938)^{* *}$ & $(-14.5075)^{*}$ & $(0.1174)$ & *** & $I(0)$ & patper & $\mathrm{N}$ & $(-4.6017) *$ & $(-6.1846) *$ & $(0.3004) *$ & $I(0)$ \\
\hline ipr & D & $(-4.7195) *$ & $(-4.7167)^{*}$ & $(0.1212)$ & *** & $I(1)$ & patsol & D & $(-4.8607)^{*}$ & $(-4.8607)^{*}$ & $(0.1366) * * *$ & * $I(1)$ \\
\hline ict & D & $(-6.2546) *$ & $(-6.4531) *$ & $(0.5000)$ & & $\mathrm{I}(1)$ & & & & & & \\
\hline
\end{tabular}

${ }^{a}$ Todas las variables están transformadas a logaritmos y para la prueba se considera que tienen intercepto y tendencia lineal. El nivel de significación para el rechazo de $\mathrm{H}_{0}$ es * rechazo al $1 \%$; ** rechazo al $5 \% ; y * *$ rechazo al $10 \%$. La N/D es la forma en que se probó y rechazó la no estacionariedad de la serie en el modelo. Así, una $\mathrm{N}$ implica que la variable se expresa en niveles y una d en primera diferencia. La $\mathrm{H}_{0}$ de las pruebas ADF y PP es existencia de una raíz unitaria, mientras que para la prueba KPSs es la estacionariedad de la serie.

Fuente: elaboración propia.

Se emplea el método de Johansen y Juselius (1990) para determinar el número de relaciones linealmente independientes, que permite aplicar las pruebas de la traza y del máximo valor propio. Se consideran un modelo general que incluye constante en el vector de cointegración, pero sin tendencia lineal en las variables

${ }^{8}$ Dada la sensibilidad de los resultados ante el empleo de diferentes series, se estiman modelos con subconjuntos de combinaciones de variables con variaciones en los retardos en el modelo VAR. No obstante, las ecuaciones estimadas para diferentes rezagos no generan cambios significativos en los resultados. 
en el vector de cointegración..$^{9}$ El estadístico $\lambda$-traza prueba la hipótesis nula secuencial de no existencia de cointegración $(r=0)$, a lo más un vector $(r<2)$, y así sucesivamente hasta llegar a la $H_{0}$ de máximo cuatro vectores de cointegración.

En la mayoría de las especificaciones, la $\lambda$-traza no puede rechazar la $H_{0}: r<3$, lo que implica que existe cointegración y que a lo más habrá dos relaciones estables de largo plazo. Así, en los modelos 1-3, 5, 7-11 y 16-22 hay evidencia para considerar dos vectores de cointegración. ${ }^{10}$ En las especificaciones 4 , 6 y 15 , el estadístico no rechaza la $H_{0}$ de existencia de menos de dos vectores de cointegración, pero sí rechaza la hipótesis de $r=0$, por lo que se acepta que esas especificaciones están cointegradas mediante una relación. En los modelos 12-14 existen tres vectores de cointegración (cuadro 3).

Cuadro 3. Análisis de cointegración. Prueba de la $\lambda$-traza (Principio de Pantula). Especificación del modelo a

\begin{tabular}{cccccccccc}
\hline Modelo & $\mathrm{Ho}$ & $\mathrm{Ha}$ & $\lambda$-traza & $\mathrm{vc}$ & Modelo & $\mathrm{Ho}$ & $\mathrm{Ha}$ & $\lambda$-traza & $\mathrm{vc}$ \\
\hline 1 & $\mathrm{r}<3$ & $\mathrm{r} \geq 3$ & $\mathbf{4 6 . 3 6 2}$ & 47.856 & 12 & $\mathrm{r}<4$ & $\mathrm{r} \geq 4$ & $\mathbf{2 6 . 4 2 4}$ & 29.797 \\
2 & $\mathrm{r}<3$ & $\mathrm{r} \geq 3$ & $\mathbf{4 4 . 5 2 0}$ & 47.856 & 13 & $\mathrm{r}<4$ & $\mathrm{r} \geq 4$ & $\mathbf{2 4 . 9 5 9}$ & 29.797 \\
3 & $\mathrm{r}<3$ & $\mathrm{r} \geq 3$ & $\mathbf{4 5 . 3 9 9}$ & 47.856 & 14 & $\mathrm{r}<4$ & $\mathrm{r} \geq 4$ & $\mathbf{2 8 . 8 0 0}$ & 29.797 \\
4 & $\mathrm{r}<2$ & $\mathrm{r} \geq 2$ & $\mathbf{6 7 . 6 6 0}$ & 69.819 & $15^{*}$ & $\mathrm{r}<2$ & $\mathrm{r} \geq 2$ & $\mathbf{8 7 . 7 5 1}$ & $\mathbf{8 8 . 8 0 4}$ \\
5 & $\mathrm{r}<3$ & $\mathrm{r} \geq 3$ & $\mathbf{4 4 . 2 0 3}$ & 47.856 & 16 & $\mathrm{r}<3$ & $\mathrm{r} \geq 3$ & $\mathbf{4 7 . 3 6 5}$ & 47.856 \\
6 & $\mathrm{r}<2$ & $\mathrm{r} \geq 2$ & $\mathbf{6 9 . 3 9 8}$ & 69.819 & 17 & $\mathrm{r}<3$ & $\mathrm{r} \geq 3$ & $\mathbf{4 7 . 5 4 4}$ & 47.856 \\
$7 *$ & $\mathrm{r}<3$ & $\mathrm{r} \geq 3$ & $\mathbf{5 7 . 1 9 2}$ & 63.876 & 18 & $\mathrm{r}<3$ & $\mathrm{r} \geq 3$ & $\mathbf{4 6 . 6 1 8}$ & 47.856 \\
8 & $\mathrm{r}<3$ & $\mathrm{r} \geq 3$ & $\mathbf{4 5 . 4 0 4}$ & 47.856 & 19 & $\mathrm{r}<3$ & $\mathrm{r} \geq 3$ & $\mathbf{4 7 . 8 3 6}$ & 47.856 \\
9 & $\mathrm{r}<3$ & $\mathrm{r} \geq 3$ & $\mathbf{5 3 . 5 8 6}$ & 47.856 & 20 & $\mathrm{r}<3$ & $\mathrm{r} \geq 3$ & $\mathbf{4 7 . 2 8 5}$ & 47.856 \\
10 & $\mathrm{r}<3$ & $\mathrm{r} \geq 3$ & $\mathbf{5 2 . 0 2 3}$ & 47.856 & 21 & $\mathrm{r}<3$ & $\mathrm{r} \geq 3$ & $\mathbf{3 2 . 9 0 0}$ & 47.856 \\
11 & $\mathrm{r}<3$ & $\mathrm{r} \geq 3$ & $\mathbf{4 7 . 8 2 5}$ & 47.856 & 22 & $\mathrm{r}<3$ & $\mathrm{r} \geq 3$ & $\mathbf{3 7 . 2 1 1}$ & 47.856 \\
\hline
\end{tabular}

a Todos los modelos se especifican con una constante en el vector de cointegración, pero sin tendencia lineal en las variables en niveles. El número de rezagos óptimo para todos los modelos es uno. Los valores mostrados son los resultados de la prueba de la traza después de ajustarse por muestra pequeña tal como lo sugieren Cheung y Lai (1993). Este ajuste se obtiene al multiplicar el valor de la prueba de la traza por el factor $(t-p k) / t$; donde $t$ es el número de datos, $p$ es el número de variables en el sistema y $k$ es el número de rezagos incluidos. vc es el valor crítico al 0.05 .

*Incluye tendencia lineal entre las variables pero no en el vector de cointegración

Fuente: elaboración propia.

${ }^{9}$ Se prueban dos especificaciones alternativas: la primera incluye tendencia lineal en las variables pero no el vector de cointegración y la segunda considera la situación contraria. Sin embargo, con la excepción de dos modelos, todos deben especificarse como la primera propuesta.

${ }^{10}$ Las especificaciones estimadas del modelo general y las variables en cada una se presentan en el cuadro 3. 
En los casos con más de un vector de cointegración se normalizó el vector "económico" para obtener la ecuación de exportaciones. Las estimaciones de los parámetros de cointegración aparecen en el cuadro 4. Las elasticidades se leen directamente de los coeficientes estimados. No todos los parámetros estimados en cualquiera de las especificaciones son estadísticamente significativos; de hecho, sólo para cuatro todas las variables son importantes en la determinación de las exportaciones $(8,15,17$ y 22). Además, únicamente la especificación 8 tiene todos los parámetros con los signos correctos. Esto sugiere que existe complementariedad en términos económicos entre las especificaciones planteadas; por lo que para explicar las exportaciones se necesita considerar todas (con parámetros significativos).

En términos generales, las importaciones manufactureras totales de EUA parecen determinantes de las exportaciones manufactureras mexicanas en el largo plazo (de es significativa en 15 de 22 especificaciones), además, tiende a afectarlas favorablemente (en 10 de éstas tiene signo positivo). De igual modo, la capacidad de producción es un factor persistente en las exportaciones hacia EUA (significativo en 17 de 22 especificaciones), aunque no es claro el signo de la relación. Adicionalmente, la estructura no competitiva de las manufactureras mexicanas influye regularmente en $x$, aunque en menor medida de lo esperado (significativo sólo en $68 \%$ de las especificaciones). Si bien en la mayoría de las especificaciones donde $i l$ es significativo impacta de manera negativa en $x$, tiende a ser un determinante importante cuando los indicadores de patentes y de ID son significativos (cuadro 4).

Los precios relativos parecen tener mayor alcance; en $91 \%$ de las especificaciones curl explica estadísticamente a $x$, lo que permite afirmar que la competitividad precio es un determinante sistemático. Por último, las capacidades tecnológicas también determinan sistemáticamente a las exportaciones (en el $72 \%$ de los casos innov es significativa). Tal como se esperaba, las capacidades tecnológicas tienden a afectar con signo positivo a las exportaciones; cuando tienen el signo "correcto" lo hacen de forma considerable (regularmente con proxies de patentes e ID).

Asimismo, en el largo plazo el gasto en ID del sector manufacturero como proporción del PIB agregado (iidl) y los gastos rezagados en ID (iid2rl e iid2r2), son determinantes de $x$. En contraste, el acervo de ID en el sector manufacturero (iid3) no es significativo, al tiempo que el gasto en ID manufacturero (iid2) afecta negativamente. La idea que subyace en esto es que importa más el nivel de gasto en relación con las necesidades o tamaño de la economía que 
el nivel absoluto de esas inversiones. También, el que iid2 mantenga una relación negativa con $x$ sugiere que se necesita tiempo para obtener resultados en términos de exportaciones, siendo inicialmente contraproducentes, quizás por la limitada productividad de esos esfuerzos. Al mismo tiempo, el impacto favorable de ID se complementa con el poder de mercado que disfrutan las empresas exportadoras dentro del mercado mexicano.

Cuadro 4. Vectores de cointegración para las exportaciones manufactureras mexicanas (1985-2006)

\begin{tabular}{|c|c|c|c|c|c|c|c|c|c|c|c|c|c|c|c|}
\hline Esp/Var & meu & curl & ivfp & il & iid1 & iid2 & iid3 & $h$ & $k h$ & kh1 & kh2 & at & atr1 & iproc & iprod \\
\hline 1 & $1.19 *$ & $-3.86 *$ & $-2.91 *$ & $0.48^{* *}$ & $-1.36^{*}$ & & & & & & & & & & \\
\hline 2 & $-4.71^{*}$ & $7.09 *$ & 0.37 ** & * 20.17* & & $2.63^{*}$ & & & & & & & & & \\
\hline 3 & $-2.61 *$ & $-3.43^{*}$ & $-6.37 *$ & $-22.76^{*}$ & & & $-0.19 * *$ & & & & & & & & \\
\hline 4 & $0.09 * *$ & $8.56 *$ & $4.08^{*}$ & $2.63^{* *}$ & & & & 0.14 ** & & & & & & & \\
\hline 5 & -2.34 ** & $12.63^{*}$ & 9.07 * & $1.03 * *$ & & & & & $2.85^{\star}$ & & & & & & \\
\hline 6 & $-2.39 *$ & $9.36^{*}$ & $6.99 *$ & -0.08 ** & & & & & & $2.52^{*}$ & & & & & \\
\hline 7 & -0.91 ** & $7.70 *$ & $4.76^{*}$ & $-2.47 * *$ & & & & & & & $0.56^{*}$ & & & & \\
\hline 8 & $-4.67^{\star}$ & $-1.24 *$ & $-12.81 *$ & $36.53^{*}$ & & & & & & & & $6.38^{*}$ & & & \\
\hline 9 & $-3.25^{*}$ & $-3.17^{*}$ & $-4.91 *$ & 18.81 * & & & & & & & & & 1.36 ** & & \\
\hline 10 & $-1.08^{*}$ & $1.42 *$ & $0.45^{\text {** }}$ & * 13.14 * & & & & & & & & & & $-1.98^{*}$ & \\
\hline 11 & $-0.51^{*}$ & 0.31 ** & * 1.01 * & $5.75 *$ & & & & & & & & & & & -1.29 * \\
\hline Esp/Var & meu & curl & ivfp & il & patsol & patcon & patcon 1 & patper & $r i$ & spmxeu & ict & $i p r$ & skteu & $i i d 2 r 1$ & iid2r2 \\
\hline 12 & $1.96 *$ & $8.72 *$ & $2.83^{* *}$ & $-1.39 * *$ & -0.67 ** & & & & & & & & & & \\
\hline 13 & $-1.17 * *$ & $-11.18 *$ & $-9.90 *$ & $-3.69 *$ & & $-1.11 *$ & & & & & & & & & \\
\hline 14 & $0.78^{\star}$ & $-4.49 * *$ & $-3.48^{*}$ & $4.94 *$ & & & $-2.09 *$ & & & & & & & & \\
\hline 15 & $-3.29 *$ & $17.06^{*}$ & $7.98^{*}$ & $9.43^{*}$ & & & & $0.53^{*}$ & & & & & & & \\
\hline 16 & $1.94^{*}$ & $8.89 *$ & $2.45^{* *}$ & * 0.26 ** & & & & & -0.45 ** & & & & & & \\
\hline 17 & $-26.38^{*}$ & $-12.57^{*}-$ & $-64.75^{*}$ & $-21.51^{*}$ & & & & & & $44.68^{*}$ & & & & & \\
\hline 18 & $-0.75 * *$ & $7.54^{\star}$ & $7.98^{*}$ & $4.52 *$ & & & & & & & $-3.60^{*}$ & & & & \\
\hline 19 & $2.15^{*}$ & 9.95* & 3.47 ** & $-0.58 *$ & & & & & & & & $0.52^{*}$ & & & \\
\hline 20 & $1.55^{* *}$ & $24.35^{\star}$ & $15.84 *$ & $-17.29 *$ & & & & & & & & & $2.92 * *$ & & \\
\hline 21 & $-0.80 * *$ & $-10.79 *$ & $-9.74^{\star}$ & $17.98^{*}$ & & & & & & & & & & $-2.46^{*}$ & \\
\hline 22 & $-1.72 *$ & -5.51 * & $-6.56^{\star}$ & $10.73^{*}$ & & & & & & & & & & & $-0.56^{*}$ \\
\hline
\end{tabular}

* Significativas al 99 y $90 \%$ de confianza, respectivamente. Esp: especificación, Var: variable. El nivel de rezago óptimo para cada especificación es uno. Se presentan los resultados del vector económico normalizado.

Fuente: elaboración propia.

Una posible explicación de los diferentes efectos de la tecnología (cambio de signos en las especificaciones) en las exportaciones es que detrás de los datos agregados existen empresas de diferentes tamaños, capacidades y recursos que enfrentan distintos grados de competencia, en el mercado nacional e internacional, por lo que la heterogeneidad implícita de éstas provoca que tengan distintos grados de asimilación de la tecnología y, por tanto, que existan diferencias en la aplicación de ésta en los procesos de exportación. En otras palabras, los 
efectos de las características particulares de las empresas en el desempeño exportador dependen del contexto específico de cada una (Sousa et al., 2008). ${ }^{11}$

Además, cuando se prueba directamente la importancia del capital humano mediante las proxies consideradas se obtiene sistemáticamente una relación negativa. Es decir, a mayor capital humano menos exportaciones, lo que puede deberse a que las mayores capacidades y conocimientos se orientan más a la producción para el mercado interno que al sector exportador, idea que se refuerza con la significación de $c p 1$ en esos mismos modelos. Aunado a lo anterior, la baja productividad del trabajo calificado en el total del trabajo manufacturero $(k h)$ y específicamente de científicos e ingenieros en el total del trabajo en la manufactura $(k h l)$ puede deberse a que las actividades de ID tienen vínculos limitados con el personal calificado, es decir, en áreas de investigación intensivas en capital humano. Por tanto, como se invierte en procesos de innovación que no son realizados por personas con capacidades científicas y tecnológicas (o al menos no en alto porcentaje) se necesita de mayores gastos, tiempo, capacidades complementarias, etcétera, para que ingenieros y científicos mexicanos generen innovaciones de alcance en el largo plazo.

Asimismo, la baja productividad de $k h$ y $k h l$ genera un proceso de adaptación lento, haciendo que at sea significativa pero con impacto negativo en las exportaciones. Esto se asocia, a su vez, con la importancia de que las empresas operen en mercados imperfectos (il significativo en esa especificación), por lo que la compra (difusión) de tecnología desde el extranjero es una estrategia que siguen las empresas dentro de las manufacturas mexicanas para participar en el mercado estadounidense. Este resultado es semejante al encontrado por Eaton y Kortum (1999), quienes señalan que la importación de tecnología incorporada en las importaciones de bienes representa una ventaja para las exportaciones agregadas. Vinculado a lo anterior, las innovaciones de proceso (iproc) y producto (iprod) explican el desempeño de las exportaciones significativa y negativamente. Cuando se innova mediante importaciones de bienes de capital y/o intermedios la ventaja que pueden obtener las exportaciones parece estar restringida.

\footnotetext{
${ }^{11}$ En este sentido, debe reconocerse que el cambio de signo en la relación entre exportaciones y tecnología y el resto de las variables en las diferentes especificaciones puede deberse a que las estimaciones no controlan por sector industrial ni por años clave. Si se consideraran estos aspectos, los resultados podrían modificarse puesto que al diferenciar por intensidades tecnológicas sectoriales, las industrias con mayor dinamismo tecnológico posiblemente registren elasticidades tecnológicas positivas independientemente del indicador de tecnología empleado, en contraste con las industrias menos intensivas en tecnología.
} 
También se observa que las patentes concedidas (patcon), y no las solicitadas, impactan en las exportaciones. La acumulación de ideas, medida como el acervo de patentes disponibles para la manufacturera mexicana (patcon1), al representar una mayor base de conocimiento codificado, aumenta la ventaja tecnológica de las $x$ en EUA. Esta idea es similar a la destacada por Fagerberg (1988) al indicar que existe un fuerte impacto de la tecnología (patentes) en las exportaciones. Las patentes concedidas a empresas dentro de la economía mexicana y el acervo de las mismas impactan en el desempeño exportador cuando las empresas operan bajo estructuras de competencia monopolística. Esto implica que para innovar y tener éxito en mercados internacionales se debe disfrutar de cierto poder de mercado y, al mismo tiempo, para mantener o ampliar la presencia en el mercado estadounidense se requiere de la protección de las patentes que le otorgan, en el mercado interno, una posición monopólica (temporal) en un bien.

La importancia de las patentes para las exportaciones es tal que la competitividad precio, que es significativa con regularidad, deja de serlo cuando las patentes lo son. En este sentido, se afirma que la competitividad tecnológica es de mayor relevancia para las exportaciones que la competitividad precio.

Además, patper y ri, patentes por persona y por empleado, respectivamente, son estadísticamente significativas. La primera tiene un efecto positivo en las exportaciones, resultado congruente con lo señalado por Narula y Wakelin (1998). Sin embargo, se estima un signo negativo en la relación entre ri y $x$, que puede deberse a la limitación de la variable, esto es, al considerar el empleo total y no el calificado como lo realizan (Papanastassiou y Pearce, 1990). Por último, se aprecia que el índice de competitividad tecnológica (ict) afecta favorablemente a $x$, acorde con los resultados de Madsen (2007). Las patentes concedidas en México tienen proporcionalmente más efecto en las ventas externas que el resto de las patentes mundiales sobre las exportaciones de competidores a ese mismo mercado, confirmándose así la importancia de los derechos exclusivos temporales otorgados por las patentes para la producción, venta o distribución de una innovación, que repercute en las exportaciones. Sin embargo, si bien las patentes estimulan las exportaciones no son la base - de acuerdo con el coeficiente asociado al índice relativo de producción de patentes (ipr)- para impulsarlas dentro del mercado de EUA.

Por último, para determinar la validez de las afirmaciones anteriores, se probó que los residuos del VAR cumplan con las condiciones para aceptar las relaciones de cointegración, verificando que las especificaciones cumplan los supuestos básicos de independencia serial, normalidad, homoscedasticidad y 
estacionariedad. Los resultados de las pruebas evidencian que, para las 22 diferentes especificaciones, los residuos se comportan bien al ser ruido blanco. En consecuencia, se confirma la existencia de alguna relación de largo plazo entre las series. ${ }^{12}$

Una vez identificada la relación de largo plazo, se continúa con el estudio de las relaciones dinámicas entre las series. Así, dado que se demostró que existe al menos una relación de cointegración, es posible especificar un VAR restringido conocido como VECM (vector de corrección de errores) para modelar las relaciones de corto y largo plazos para las seis variables comprendidas en el sistema en cada especificación. Por tanto, se estimó un modelo VECM, con $k^{*}=1$ de acuerdo con los criterios de AIC y LR, de la forma $\Delta y_{t}=\alpha w_{t-1}+C+A_{t} \Delta y_{t-1}+u_{\mathrm{t}}$, con $w_{t-1}=\beta^{\prime} \cdot X_{t-1}$, que representa las combinaciones lineales de cointegración, mientras que $u_{\mathrm{t}}$ son los términos de error estacionarios, $C$ es un vector de constantes de dimensión $(6 \times 1), A_{l}$ es una matriz de coeficientes de dimensión $(6 \times 22)$ y $\Delta y_{t-1}$ es el vector de variables endógenas en primeras diferencias retardadas un periodo.

Se estimaron los coeficientes de ajuste del sistema al equilibrio de largo plazo, que determinan la rapidez con la que este equilibrio se restablece después que ha ocurrido un choque e indican cuáles variables son las que realizan esta corrección. De los coeficientes de velocidad de ajuste asociados a cada vector de cointegración, que dependiendo la especificación varían entre uno y tres vectores, se concluye que todas las series ajustan sus propios vectores ya que ante un desequilibrio dentro de cualquier vector son las primeras diferencias de $x, m e u$, curl, ivfpln, il y de cualquiera de las proxies de tecnología, las que se ajustan para regresar el sistema al equilibrio.

Sin embargo, en el cuadro 5 sólo se presentan las ecuaciones de corrección de errores con el mayor número de variables significativas y con el signo "correcto" para cada una de las especificaciones. Así, por ejemplo, en la especificación 1 se observa que los términos de corrección de error asociados a $D(\mathrm{meu})$,

${ }^{12}$ Asimismo, se requiere que exista estabilidad de los parámetros del modelo estimado para que la inferencia sea válida. Por ende, si la prueba consistió en probar si durante 1985-2006 ocurrió algún evento estadísticamente significativo que provocara inestabilidad (cambio estructural) en las variables, a partir de la metodología de Gregory y Hansen (1986) se examina la presencia de relaciones de cointegración bajo posibles cambios de régimen (asumiendo que existe un cambio de estructura en fecha desconocida). Las pruebas InADF y MeanADF señalan, para todos los casos, que a pesar de existir cambio estructural en 1995 -menos para la especificación 15 donde el cambio de estructura es en 1996-, hay evidencia de cointegración, es decir, se mantiene la estabilidad paramétrica en la relación de largo plazo. 
$D($ curl) y $D$ (iidl) tienen signos negativos, lo que sugiere que éstas contribuyen a la restauración de la relación de equilibrio en el largo plazo, cuando éste es perturbado por un choque inesperado en el corto plazo. De este modo, en la mayoría de las especificaciones hay dos o más variables que colaboran al ajuste ( $71 \%$ de los casos), señalando la importancia de la dinámica de corto plazo para explicar el comportamiento de las exportaciones manufactureras. En seis especificaciones una sola variable es la que corrige el desequilibrio $(4-6,13,15$ y 22$)$ y en el caso 19 no hay ajuste.

Cuadro 5. Vectores de correccion de errores de las exportaciones manufactureras mexicanas (1985-2006)

\begin{tabular}{|c|c|c|c|c|c|c|c|c|c|c|c|c|c|c|c|c|c|}
\hline Esp / Var & $x$ & meu & curl & $i v f p$ & il & iid1 & iid2 & iid3 & $h$ & $k h$ & kh1 & kh2 & at & atr1 & iproc & iprod & $R^{2}$ \\
\hline 1 & -0.065 & $-0.047^{*}$ & $-0.015^{\star}$ & 0.016 & 0.002 & $-0.105^{*}$ & & & & & & & & & & & 0.73 \\
\hline 2 & 0.135 & -0.007 & $-0.048^{*}$ & -0.011 & -0.003 & & $-0.225^{*}$ & & & & & & & & & & 0.71 \\
\hline 3 & -0.207 * & $-0.127^{*}$ & $-0.055^{\star}$ & 0.003 & 0.017 & & & $-0.146^{*}$ & & & & & & & & & 0.69 \\
\hline 4 & -0.032 & 0.005 & -0.030 * & 0.005 & 0.033 & & & & 0.003 & & & & & & & & 0.43 \\
\hline 5 & -0.110 & $-0.187^{*}$ & $-0.022 *$ & 0.015 & 0.034 & & & & & 0.001 & & & & & & & 0.63 \\
\hline 6 & 0.005 & 0.026 & $-0.019 *$ & 0.003 & 0.027 & & & & & & -0.009 & & & & & & 0.38 \\
\hline 7 & 0.104 & -0.452 & $-0.059 *$ & -0.037 & -0.008 & & & & & & & $0.722^{*}$ & & & & & 0.58 \\
\hline 8 & 0.139 & 0.013 & 0.003 & $-0.078^{*}$ & $-0.042 *$ & & & & & & & & $-0.094^{*}$ & & & & 0.62 \\
\hline 9 & 0.016 & 0.004 & 0.003 & 0.063 & -0.027 * & & & & & & & & & $-0.075^{*}$ & & & 0.62 \\
\hline 10 & -0.227 & 0.011 & $-0.169 *$ & 0.006 & 0.184 & & & & & & & & & & $-1.118^{*}$ & & 0.67 \\
\hline 11 & $-0.208^{*}$ & -0.019 & $-0.022^{*}$ & 0.013 & $-0.044 *$ & & & & & & & & & & & -0.109 * & 0.56 \\
\hline Esp / Var & $x$ & meu & curl & $i v f p$ & il & patsol & patcon $p$ & patcon 1p & patper & $r i$ & spmxeu & ict & ipr & skteu & $i i d 2 r 1$ & $i i d 2 r 2$ & $R^{2}$ \\
\hline 12 & -1.599 & $-1.021 *$ & $-0.439 *$ & -0.171 & 0.161 & -1.139 & & & & & & & & & & & 0.65 \\
\hline 13 & -0.006 & -0.043 & $-0.227^{*}$ & -0.102 & 0.163 & & 0.261 & & & & & & & & & & 0.45 \\
\hline 14 & $-0.513^{*}$ & $-0.338^{*}$ & $-0.149 *$ & 0.003 & 0.023 & & & $-2.051^{*}$ & & & & & & & & & 0.71 \\
\hline 15 & -0.008 & 0.009 & $-0.021 *$ & -0.002 & 0.023 & & & & -0.065 & & & & & & & & 0.43 \\
\hline 16 & -0.043 & 0.001 & $-0.020^{*}$ & $-0.098^{*}$ & 0.081 * & & & & & 0.039 & & & & & & & 0.46 \\
\hline 17 & -0.030 & -0.036 * & -0.004 & 0.007 & $-0.013 *$ & & & & & & 0.010 & & & & & & 0.45 \\
\hline 18 & 0.001 & -0.069 & $-0.058^{*}$ & 0.004 & 0.001 & & & & & & & $-0.131 *$ & & & & & 0.49 \\
\hline 19 & -0.047 & 0.004 & -0.016 & 0.040 & 0.019 & & & & & & & & -0.043 & & & & 0.39 \\
\hline 20 & -0.027 & -0.019 & $-0.017 *$ & 0.008 & -0.510 * & & & & & & & & & $-0.029 *$ & & & 0.56 \\
\hline 21 & 0.056 & 0.027 & -0.007 & -0.003 & -0.031 * & & & & & & & & & & $-0.179 *$ & & 0.54 \\
\hline 22 & 0.082 & 0.022 & 0.030 & -0.002 & -0.027 * & & & & & & & & & & & -0.024 & 0.47 \\
\hline
\end{tabular}

*Significativas al $95 \%$ de confianza. En todos los casos se muestran los coeficientes dentro del vector de corrección de errores que son significativos y que tienen el signo correcto, esto es, que representan una relación de largo plazo estable. Todas las variables están expresadas en primeras diferencias.

Fuente: elaboración propia.

Las variables con mayor contribución a la eliminación del desajuste son curl (64\% de los casos), innov (55\%) e il (32\%), en consecuencia, las desviaciones a partir de la tendencia de largo plazo compartida por las seis variables cointegradas se describen mejor como movimientos transitorios de estas tres series. No obstante, la respuesta es lenta, lo que se aprecia por la magnitud de los 
coeficientes de ajuste. En particular, los coeficientes más altos están en las especificaciones 20 y 14 y corresponden a $i l$ y meu, que corrigen 51 y $34 \%$ del desequilibrio por año, respectivamente. Considerando las especificaciones en las que una variable dada contribuye a la corrección, las que lo hacen con mayor velocidad son innov (coeficiente promedio 0.30), mеи (0.15) y curl (0.08). Este análisis individual revela que el VECM posee en conjunto una dinámica de corrección complementaria.

Asimismo, prácticamente todos los coeficientes de largo plazo son mayores a los de corto, excepto para kh2 y patsol, lo que sugiere la existencia de una oferta exportadora de mayor magnitud en el horizonte largo. En particular, se tiene que el parámetro de la variable innov aumenta cuando se pasa del corto al largo plazo. Esto es, en general, que la tecnología favorece a las exportaciones manufactureras, porque las actividades de ID permiten obtener innovaciones incrementales en productos o procesos en el largo plazo y no en el corto. En consecuencia, los productos exportados encuentran espacio en el mercado de EUA. Estas innovaciones evitan una contracción del nivel de ventas en el exterior al alargar el ciclo de vida de los productos, impidiendo que se conviertan rápidamente en "obsoletos".

Por otro lado, se practican pruebas de exclusión y exogeneidad individual de las variables en el VECM. La exogeneidad de una variable en un sistema implica que en el análisis de esa relación se consideran como dados los valores de la variable exógena, por lo que no es necesario modelarla explícitamente (Galindo, 1997). De igual forma, se debe probar si todas las variables son importantes dentro del vector de cointegración y, así, estipular si las variables pueden ser excluidas de la relación.

El cuadro 6 presenta los resultados de la prueba de exogeneidad débil, que rechaza la nula con un nivel de significación de $5 \%$ para todas las variables en 6 especificaciones $(1,5,8,10,13$ y 18); para todas las variables, excepto una, en 9 especificaciones $(6,9,11,12,15,17,19,20$ y 22) y para todas las variables, excepto dos, en 7 casos $(2,3,4,7,14,26$ y 21); $x$ e ivfp son siempre exógenas débiles; meu e $i l$ no son exógenas en 4 y 5 especificaciones, respectivamente; mientras que innov no lo es en seis casos (iid3, iprod, patcon1, spmxeu, iid2rl e iid2r2). Considerando todas las especificaciones, se tiende a aceptar, en general, la exogeneidad débil de todas las variables (en menor medida para curl). Los resultados permiten concluir que todas las variables son causadas débilmente por las restantes cinco, lo que confirma la intuición de que las exportaciones son una variable endógena. ${ }^{13}$

${ }^{13}$ Se estima además el estadístico $\chi^{2}$ para 6 subperiodos (el año inicial de cada subperiodo es 1985 y final 2001, 2002, 2003, 2004, 2005 y 2006, respectivamente) estableciéndose restricciones 
De igual forma, el cuadro 6 muestra los resultados de la prueba de exclusión para cada especificación. Se observa que existe evidencia estadística para rechazar la hipótesis de exclusión individual para todas las variables en 13 de las 22 especificaciones, es decir, todas las variables del sistema son imprescindibles en el vector de corrección; meu puede ser eliminada del VECM en seis casos $(4,5$, $15,16,18$ y 20), mientras que innov no integra la relación de corto plazo en tres especificaciones ( $h$, patper, ipr) (véase cuadro 6).

\section{Cuadro 6. Pruebas de exogeneidad débil y exclusión de VECM}

\begin{tabular}{|c|c|c|c|c|c|c|c|c|c|c|c|c|c|c|c|c|c|c|c|c|c|c|}
\hline \multirow{2}{*}{$\begin{array}{c}\text { Esp } \\
\text { Variable }\end{array}$} & \multicolumn{2}{|c|}{1} & \multicolumn{2}{|c|}{2} & \multicolumn{2}{|c|}{3} & \multicolumn{2}{|r|}{4} & \multicolumn{2}{|r|}{5} & \multicolumn{2}{|c|}{6} & \multicolumn{2}{|c|}{7} & \multicolumn{2}{|c|}{8} & \multicolumn{2}{|c|}{9} & \multicolumn{2}{|c|}{10} & \multicolumn{2}{|c|}{11} \\
\hline & Exc & Exo & Exc & Exo & Exc & Exo & Exc & Exo & Exc & Exo & Exc & Exo & Exc & Exo & Exc & Exo & Exc & Exo & Exc & Exo & Exc & Exo \\
\hline$x$ & 1.54 & 10.66 & 1.16 & 7.75 & 3.97 & 22.65 & 0.36 & $3.76 *$ & * 2.11 & 15.07 & 0.01 & 12.58 & 0.48 & 11.63 & 1.57 & 16.09 & 4.35 & 17.06 & 1.06 & 22.09 & 1.67 & 11.56 \\
\hline meu & 3.55 & 9.04 & 11.08 & * 14.12 & $5.90^{*}$ & *21.33 & 0.03 & $0.04 *$ & 1.25 & $3.40^{*}$ & $* 1.25$ & 8.11 & 1.90 & 18.02 & 0.31 & 35.56 & 0.54 & 13.31 & 0.83 & 8.53 & 0.55 & 9.73 \\
\hline curl & 1.29 & 20.20 & $4.89 *$ & $* 18.82$ & 0.68 & 26.26 & $9.97 *$ & 11.03 & 6.01 & 8.72 & 2.04 & 8.88 & $6.92 *$ & 22.28 & 0.99 & 9.27 & $9.10^{*}$ & 14.80 & 1.52 & $6.49 *$ & * 0.91 & 6.05 \\
\hline$i v f p$ & 2.97 & 16.03 & 0.31 & 0.13 & 0.38 & 34.38 & 0.24 & 8.76 & 1.57 & 6.59 & 0.12 & 6.69 & 2.34 & 5.91 * & *0.25 & 26.07 & 0.26 & 13.18 & 0.14 & $5.36^{*}$ & * 0.83 & 6.97 \\
\hline il & 1.27 & 4.79 & 0.30 & 14.75 & 2.51 & 63.17 & $2.43 *$ & $* 0.57$ * & 2.89 & $4.19 *$ & * 4.21 * & * 13.31 & $12.18^{*}$ & 22.77 & 0.64 & 35.50 & 0.77 & 34.45 & 2.53 & 28.95 & 1.13 & 17.26 \\
\hline iid1 & 2.96 & 18.15 & & & & & & & & & & & & & & & & & & & & \\
\hline$i i d 2$ & & & 1.85 & 14.45 & & & & & & & & & & & & & & & & & & \\
\hline iid3 & & & & & $7.03^{*}$ & 22.20 & & & & & & & & & & & & & & & & \\
\hline$h$ & & & & & & & 0.034 & $1.96^{*}$ & & & & & & & & & & & & & & \\
\hline$k h$ & & & & & & & & & 0.391 & $2.99 *$ & & & & & & & & & & & & \\
\hline kh1 & & & & & & & & & & & 0.225 & 57.54 & & & & & & & & & & \\
\hline kh2 & & & & & & & & & & & & & 4.625 & 16.24 & & & & & & & & \\
\hline at & & & & & & & & & & & & & & & 1.24 & 35.34 & & & & & & \\
\hline atr1 & & & & & & & & & & & & & & & & & 0.025 & 8.25 & & & & \\
\hline iproc & & & & & & & & & & & & & & & & & & & 2.63 & 26.04 & & \\
\hline iprod & & & & & & & & & & & & & & & & & & & & & $3.09 * *$ & * 18.83 \\
\hline Esp & & 12 & 1 & 3 & 1 & 4 & & 15 & & 16 & 1 & 17 & & 18 & 1 & 19 & 2 & 0 & 2 & 1 & 22 & 2 \\
\hline Variable & Exc & Exo & Exc & Exo & Exc & Exo & Exc & Exo & Exc & Exo & Exc & Exo & Exc & Exo & Exc & Exo & Exc & Exo & Exc & Exo & Exc & Exo \\
\hline$x$ & 3.35 & 11.48 & 0.78 & 13.56 & 3.91 & 25.40 & 1.58 & 11.67 & 0.45 & 13.16 & 0.54 & 9.48 & 0.15 & 10.54 & 0.47 & $3.36^{*}$ & $* 0.69$ & 8.79 & 4.33 & 10.06 & 1.48 & 9.97 \\
\hline meu & 2.82 & 2.90 & 1.76 & 3.09 & $9.76^{*}$ & 9.38 & 1.87 & $3.67 *$ & $11.10^{*}$ & * $3.77 *$ & * 3.40 & 7.60 & 1.37 & $0.78^{*}$ & 0.02 & 7.61 & 2.23 & $2.95^{*}$ & 2.81 & 24.10 & 3.55 & 10.58 \\
\hline curl & 2.01 & 11.20 & 4.11 & 14.22 & 1.92 & 21.44 & $9.07 *$ & 4.32 & $4.25^{*}$ & 6.43 & 2.86 & 7.31 & 1.32 & 9.87 & $8.55^{*}$ & $5.76^{*}$ & $* 2.53$ & 16.28 & $5.77^{*}$ & $\approx 28.99$ & 1.66 & 22.47 \\
\hline ivfp & 5.02 & 12.06 & 5.14 & 17.36 & 3.29 & 17.69 & 4.35 & 5.27 & 0.01 & 9.95 & 0.85 & 14.98 & 0.07 & 9.55 & 4.69 & 9.90 & 0.97 & 7.04 & 0.53 & 14.98 & 0.53 & 20.48 \\
\hline il & 8.00 * & * 6.83 & 3.06 & 5.78 & 1.29 & 29.59 & 1.14 & 16.12 & 3.11 & 3.07 * & * 1.97 & 5.23 & 1.24 & 6.96 & 0.61 & 8.14 & $8.49 *$ & 21.50 & 4.12 & 39.57 & 1.32 & 24.87 \\
\hline patsol & 0.93 & 7.85 & & & & & & & & & & & & & & & & & & & & \\
\hline patcon & & & 3.00 & 7.94 & & & & & & & & & & & & & & & & & & \\
\hline patcon1 & & & & & $11.75^{\star}$ & +30.63 & & & & & & & & & & & & & & & & \\
\hline patper & & & & & & & 0.83 & $3.68^{* *}$ & & & & & & & & & & & & & & \\
\hline$r i$ & & & & & & & & & 0.26 & 6.42 & & & & & & & & & & & & \\
\hline spmxeu & & & & & & & & & & & $5.17 *$ & * 15.48 & & & & & & & & & & \\
\hline ict & & & & & & & & & & & & & 2.89 & 6.93 & & & & & & & & \\
\hline$i p r$ & & & & & & & & & & & & & & & 0.27 & $5.84^{* *}$ & & & & & & \\
\hline skteu & & & & & & & & & & & & & & & & & 4.95 & 6.83 & & & & \\
\hline$i i d 2 r 1$ & & & & & & & & & & & & & & & & & & & $22.94^{\star}$ & 35.96 & & \\
\hline$i i d 2 r 2$ & & & & & & & & & & & & & & & & & & & & & 7.61 * & 8.93 \\
\hline
\end{tabular}

* y ** indican que la variable no es exógena débil al 1\% y 5\% de significación, respectivamente, de acuerdo al estadístico LR de máxima verosimilitud y al $p$-value asociado. $\mathrm{H}_{0}$ no exogeneidad débil. $\mathrm{H}_{0}$ : exclusión individual. Todas las variables están expresadas en primeras diferencias. Esp: especificación.

Fuente: elaboración propia.

de exogeneidad débil. La prueba consistentemente no rechaza $\mathrm{H}_{0}$ (exogeneidad individual) para todas las variables, excepto para curl. 
Considerando las pruebas de exogeneidad débil y de exclusión, se acepta como adecuado el modelo estimado para las exportaciones mexicanas. El VECM satisface todas las pruebas estadísticas básicas, incluyendo el carácter exógeno de la variable innov (en la mayoría de sus proxies); sin embargo, el hecho de que no se presente esta condición en algunos modelos limita el alcance predictivo del modelo general estimado, lo que debe considerarse al momento de la elaboración de política comercial. ${ }^{14}$

Por último, dado que en las diferentes especificaciones se confirmó la existencia de relaciones estables de largo plazo, en el sistema formado por exportaciones, demanda extranjera, capacidad de producción, precios relativos, competencia monopolística y capacidades tecnológicas, se determinó también la dirección de la causalidad entre las variables, aplicando la prueba de causalidad de Granger. La causalidad en el sentido de Granger implica que los valores presentes y pasados de $x$ ayudan a predecir mejor los valores de $y$. Al emplearse el VECM se puede establecer la causalidad y la dirección de la misma, con la prueba de Wald, variable por variable (cuadro 7).

En general, las estimaciones permiten comprobar la existencia de una relación causal que va de meu, curl, ivfp e $i l$ a $x$ (cuadro 7). Al considerar las variables tecnológicas, se tiene que para la mayoría de las relaciones la dirección de causalidad es de éstas hacia las exportaciones. Basados en los valores de $p$, se rechaza la hipótesis nula de no causalidad en 19 de las 24 relaciones entre variables, lo que sugiere que sistemáticamente el sentido de la causalidad es hacia las exportaciones..$^{15}$ Lo anterior significa que demanda extranjera, precios relativos, capacidad de producción, competencia monopolística y capacidades tecnológi-

\footnotetext{
${ }^{14}$ Se verificó que los residuos del VECM cumplan con independencia serial, normalidad, homoscedasticidad y estacionariedad. De acuerdo con las pruebas de raíz unitaria ADF, PP y KPSS, los residuos del VEC son estacionarios al 99\% de confianza en niveles, para 20 de las especificaciones y para 2 lo son en primeras diferencias. La prueba Breusch-Godfrey (LM) examina la existencia de correlación de orden $(h)$ en los residuos; no hay evidencia de autocorrelación en ninguna especificación, ya que los valores de $p$ muestran que los residuos son ruido blanco desde el primer retardo. El test de normalidad no rechaza la normalidad conjunta de los residuos para ninguna especificación, ya que la probabilidad es mayor a $0.05 \%$.

Para que el VECM pueda aceptarse como robusto debe cumplir con la no presencia de heteroscedasticidad condicional, que se prueba con el estadístico LM-ARCH; se acepta, en todos los casos, la nula de no existencia de heteroscedasticidad condicional autorregresiva (valores de $p>0.05 \%$ ).

${ }^{15}$ La prueba de causalidad de Granger se aplicó además para determinar si la dirección de causalidad era de $x$ hacia las demás variables. En 12 relaciones no se rechazó la nula (menos de la mitad del total), por lo que, en general, parece que la causalidad se mueve en una sola dirección, esto es, desde innov, il, ivfp, curl y meu a $x$.
} 
cas, tienen un efecto positivo en las ventas al extranjero. En consecuencia, se confirma la hipótesis que las exportaciones mexicanas hacia EUA son impulsadas por factores tecnológicos y de organización industrial.

\section{Cuadro 7. Prueba de causalidad de Granger para exportaciones manufactureras} mexicanas y variables independientes*

\begin{tabular}{|c|c|c|c|c|c|c|c|}
\hline Variables independientes & Prob & $\mathrm{H}_{0}$ & Caus & & & & \\
\hline meu & 0.044 & $\mathrm{RH}_{0}$ & $m e u \rightarrow x$ & & & & \\
\hline curl & 0.044 & $\mathrm{RH}_{0}$ & curl $\rightarrow x$ & & & & \\
\hline$i v f p$ & 0.031 & $\mathrm{RH}_{0}$ & $i v f p \rightarrow x$ & & & & \\
\hline il & 0.032 & $\mathrm{RH}_{0}$ & il $\rightarrow x$ & & & & \\
\hline Variables tecnológicas & Prob & $\mathrm{H}_{0}$ & Caus & Variables tecnológicas & Prob & $\mathrm{H}_{0}$ & Caus \\
\hline iid1 & 0.048 & $\mathrm{RH}_{0}$ & iid1 $\rightarrow x$ & patsol & 0.191 & $\mathrm{NRH}_{0}$ & \\
\hline iid2 & 0.011 & $\mathrm{RH}_{0}$ & $i i d 2 \rightarrow x$ & patcon & 0.170 & $\mathrm{NRH}_{0}$ & \\
\hline iid3 & 0.181 & $\mathrm{NRH}_{0}$ & & patcon1 & 0.007 & $\mathrm{RH}_{0}$ & patcon $1 \rightarrow x$ \\
\hline$h$ & 0.002 & $\mathrm{RH}_{0}$ & $h \rightarrow x$ & patper & 0.189 & $\mathrm{NRH}_{0}$ & \\
\hline$k h$ & 0.049 & $\mathrm{RH}_{0}$ & $k h \rightarrow x$ & ri & 0.044 & $\mathrm{RH}_{0}$ & $r i \rightarrow x$ \\
\hline kh1 & 0.014 & $\mathrm{RH}_{0}$ & $k h 1 \rightarrow x$ & spmxeu & 0.021 & $\mathrm{RH}_{0}$ & spmxeu $\rightarrow x$ \\
\hline$k h 2$ & 0.057 & $\mathrm{NRH}_{0}$ & & ict & 0.021 & $\mathrm{RH}_{0}$ & $i c t \rightarrow x$ \\
\hline at & 0.011 & $\mathrm{RH}_{0}$ & $a t \rightarrow x$ & ipr & 0.142 & $\mathrm{NRH}_{0}$ & \\
\hline at1 & 0.039 & $\mathrm{RH}_{0}$ & at1 $\rightarrow x$ & skteu & 0.042 & $\mathrm{RH}_{0}$ & skteu $\rightarrow x$ \\
\hline iproc & 0.019 & $\mathrm{RH}_{0}$ & iproc $\rightarrow x$ & $i i d 2 r 1$ & 0.045 & $\mathrm{RH}_{0}$ & $i i d 2 r 1 \rightarrow x$ \\
\hline iprod & 0.005 & $\mathrm{RH}_{0}$ & iprod $\rightarrow x$ & $i i d 2 r 2$ & 0.159 & $\mathrm{NRH}_{0}$ & \\
\hline
\end{tabular}

*Se sigue el método de Granger, por tanto lo que se muestra es el valor de la probabilidad (prob) de la prueba $\mathrm{F}$ de significación conjunta de Wald. $\mathrm{H}_{0}$ : la variable independiente no causa a la dependiente. $\mathrm{NRH}_{0}$ : no rechazo de la hipótesis nula al $5 \%$ de significación. $\mathrm{RH}_{0}$ : rechazo de la hipótesis nula al $5 \%$ de significación. Caus: causalidad.

$\mathrm{La} \rightarrow$ indica la existencia de una relación de causalidad al tiempo que muestra el sentido de ésta. La prueba se realiza con el valor estándar de dos retardos en todos los casos. El número de observaciones es 20 .

Fuente: elaboración propia.

\section{CONCLUSIONES}

El propósito de este estudio es demostrar que en el largo plazo las exportaciones mexicanas hacia EUA son impulsadas principalmente por factores tecnológicos. En particular, partiendo de un modelo de determinantes de las exportaciones, que supone que los bienes intercambiados son sustitutos imperfectos y considera elementos tanto de oferta como de demanda, se incorporaron variables tecnológicas y de estructura de mercado para identificar si éstas tienen significancia sistemática a lo largo de las diferentes estimaciones del modelo en un horizonte de tiempo 
corto y largo. Para ello, se estimó un modelo que incluye, alternativamente, 22 diferentes variables tecnológicas.

En general, los factores tecnológicos, considerando alternativamente las proxies de tecnología, en combinación con la estructura de mercado, tienen mayor impacto en la determinación de las exportaciones manufactureras de largo plazo, en comparación con la competitividad-precio. Mantener inversiones en ID durante largo tiempo, obtener patentes sistemáticamente, adquirir tecnología desde el extranjero y, en general, realizar innovaciones de proceso y/o producto, lleva en el largo plazo a mayores exportaciones. La elasticidad ingreso sugiere que los beneficios que obtienen los exportadores mexicanos de un choque positivo en el ingreso de EUA son positivos y conduce, ceteris paribus, a un aumento en las ventas en el mercado estadounidense. Además, la obtención de beneficios extraordinarios en el mercado interno, medidos por el índice de Lerner, impulsa el nivel exportado. En específico, la competencia monopolística favorece a $x$.

La capacidad de producción de las empresas mexicanas está correlacionada positivamente con las exportaciones en el largo plazo. Asimismo, los costos laborales unitarios relativos son relevantes para las ventas en el extranjero. Esto es, en la medida en que el salario por trabajador en EUA crezca en comparación con el salario pagado por los exportadores mexicanos, éstos obtienen una ventaja en términos de costos. Cabe señalar que si bien curl es estadísticamente significativa en el largo plazo tiene, en promedio, un efecto menor al de casi cualquier otra variable.

De esta manera, el análisis se centra en las capacidades tecnológicas desarrolladas por la industria manufacturera mexicana como un mecanismo para exportar a Estados Unidos. En general, los gastos en ID guardan en el largo plazo un efecto positivo relativamente bajo; en promedio, menor a la unidad para todas las estimaciones, lo que permite argumentar la ineficiencia de los gastos tecnológicos de las empresas manufactureras mexicanas. Por cada nuevo millón de dólares invertidos en ID, las exportaciones crecen menos que proporcionalmente en sólo 430 mil dólares, es decir, se "pierden" 57 centavos de cada dólar de esa inversión. Surge así la hipótesis de que las empresas exportadoras crean, mediante tales gastos, una "brecha tecnología-exportación"; esto es, como se necesita un alto nivel de inversión para generar poco valor de los bienes exportables, el diferencial se "pierde", empeorando su posición competitiva en el largo plazo. Dado este argumento, la pérdida de posición de una empresa exportadora se debe a la insuficiencia de recursos y capacidades tecnológicas eficientes que permitan asi- 
milar eficientemente los nuevos conocimientos y tecnologías para superar los distintos obstáculos en el proceso de exportación.

La idea de ineficiencia de la ID en la relación de largo plazo con las exportaciones se asocia con el ciclo de vida del producto. El monto y tipo de ID en el sector manufacturero se realiza en etapas "atrasadas" o "básicas" del ciclo, siendo un obstáculo para que las empresas amplíen sus actividades en los mercados de exportación, puesto que el ritmo de obsolescencia de los productos tecnológicos es alto. De esta manera, siguiendo a Harris y Li (2009), las empresas enfrentan el desafío de aprender, compartir y asimilar el nuevo conocimiento para superar las barreras a las exportaciones. Al mismo tiempo, establecer lazos de cooperación más fuertes con empresas tanto dentro de la misma industria como de otros sectores permitiría que se desarrollen las capacidades de innovación-producción-exportación (Lefebvre et al., 1998).

Una de las razones de este efecto ineficiente es que las actividades formales de ID de las empresas mexicanas sólo representan una pequeña parte de la creación de capacidades tecnológicas requeridas para la actividad económica, esto es, se encuentran por debajo de la proporción necesaria (OCDE, 2007). Además, como lo señala Romijn (1997), estas actividades consisten fundamentalmente en la compra de tecnología básica de orientación práctica a algún proveedor extranjero, por lo que la innovación se centra en pequeñas modificaciones para adaptarlas a las condiciones locales, en la puesta en operación, reparación, mantenimiento y, en el mejor de los casos, en el "ajuste" de esta tecnología para la venta a otras empresas. ${ }^{16}$ En consecuencia, como la innovación en productos o procesos se realiza con base en los conocimientos acumulados de actividades anteriores de investigación y desarrollo (que contribuyen a aumentar el acervo de conocimientos disponible para innovaciones futuras), el nivel de innovaciones obtenidas por los gastos en ID es meramente incremental.

De igual manera, las patentes en cualquiera de sus mediciones (concedidas, acervo o flujo, por persona o dentro del índice de competitividad tecnológica) tienden a fomentar las exportaciones. Por tanto, establecer, procesos de investigación sistematizados, formales o no, dentro de las empresas exportado-

\footnotetext{
${ }^{16}$ Lo que es más, los gastos en ID en México son bajos en términos absolutos y respecto al PIB, y tienden a concentrarse en universidades, centros de investigación públicos y en pocos laboratorios privados. Esta situación, aunada al hecho de que existen políticas orientadas al impulso de sectores prioritarios, tiende a centralizar las actividades de ID en ciertas áreas, limitando los vínculos entre agentes y, por ende, la transferencia, desarrollo y aplicación del conocimiento y tecnologías.
} 
ras, es una estrategia que debería seguirse para participar en los mercados internacionales. Así, entre mayor sea la razón de patentes respecto a la población, mayor será la competitividad de un país en el mercado estadounidense, idea consistente con la propuesta de Narula y Wakelin (1998). No obstante, esta competitividad está condicionada por las patentes internacionales, esto es, comparativamente las patentes concedidas en la economía mexicana frente a las patentes de empresas competidoras en EUA parecen no representar una ventaja para acceder a ese mercado (ipr es no significativo).

En cuanto a la relación de corto plazo, para prácticamente todos los casos se estimaron coeficientes menores a los de largo plazo, implicando que la capacidad de exportación de la economía mexicana aumenta mediante procesos de aprendizaje una vez que los agentes capitalizan favorablemente la información, tecnología, infraestructura, etcétera, en el transcurso del tiempo, haciendo a las empresas más competitivas. Especialmente, como el coeficiente de las variables tecnológicas aumenta al pasar del corto al largo plazo, se afirma que el nivel de ineficiencia es mayor en el corto plazo, por lo que el learning-by-doing parece ser un mecanismo fundamental para el éxito exportador mexicano.

De este modo, mientras la inversión en capacidades tecnológicas no sea a través de proyectos de largo plazo; mientras esta inversión no sea efectivamente complementada con factores como capital humano y redes de colaboración que potencien su efecto, generando más innovaciones incrementales y algunas radicales; mientras estos gastos no conciban una diversificación de productos y mercados así como patentes y licencias de aplicación estratégica, la contribución de estas capacidades a las exportaciones mexicanas en el corto y largo plazos continuará siendo reducida y, lo que es peor, puede llevar a la marginación de estos bienes en el mercado de EUA.

\section{REFERENCIAS BIBLIOGRÁFICAS}

Amoroso, N., D. Chiquiar, N. Quella, y M. Ramos-Francia (2008), "Determinantes de la ventaja comparativa y del desempeño de las exportaciones manufactureras mexicanas en el periodo 1996-2005”, Documento de Investigación núm. 2008-1, Banco de México, México.

Bell, M, y K. Pavitt (1993), “Accumulating Technological Capability in Developing countries", Banco Mundial (comp.), Proceedings of The World Bank Annual Conference on Development Economics, Banco Mundial, Washington, páginas 257-281. 
Bernard, A., y J. Bradford (2004), "Why Some Firms Export", The Review of Economics and Statistics, Vol. 86-2, Massachusetts, pp. 561-569.

Bleany, M., y K. Wakelin (2002), "Efficiency, Innovation and Exports", Oxford Bulletin of Economics and Statistics, Vol. 64-1, Oxford, pp. 3-15.

Cotsomitis, J., C. de Bresson, y A. Kwan (1991), “A Reexamination of the Technology Gap Theory of Trade: Some Evidence from Time Series Data for OECD Countries", Review of World Economics, Vol. 127, Kiel, Alemania, pp. 792-799.

Delgado, A., J. Fariñas, y S. Ruano (2002), "Firm Productivity and Export Markets: A Nonparametric Approach", Journal of International Economics, Vol. 57-2, Madison, Wisconsin, pp. 397-422.

Dunning, J. (1995), "Reappraising the Eclectic Paradigm in an Age of Alliance Capitalism”, Journal of International Business Studies, Vol. 26-3, Lorraine, pp. 461491.

Eaton, J., y S. Kortum (1999), "International Patenting and Technology Diffusion: Theory and Measurement", International Economic Review, Vol. 40-43, Pennsylvania, pp. 537-570.

Fagerberg, J. (1996), “Technology and Competitiveness", Oxford Review of Economic Policy, Vol. 12-3, Nueva York, pp. 39-51.

(1988), "International Competitiveness", The Economic Journal, Vol. 98-3, Londres, pp. 355-374.

y B. Verspagen (2002), “Technology Gaps, Innovation, Diffusion and Transformation: An Evolutionary Approach”, Research Policy Documents, 31, Dublín, pp. 1291-1304.

Galindo, P. (1997), "El concepto de exogeneidad en la econometría moderna", Investigación Económica, Vol. 52, México, pp. 97-111.

Goldar, B. (1989), "Determinants of India's Export Performance in Engineering Products, 1960-79”, The Developing Economies, Vol. 27, Japón, pp. 3-18,

Goldstein, M., y M. Khan, (1986), "Income and Price Effects in Foreign Trade", en Jones, R., y P. Kenen (comps.), Handbook of International Economics, NorthHolland, Ámsterdam, pp. 275-286.

Graf, J. (1996), "El crecimiento de las exportaciones y el desempeño de la productividad en la industria manufacturera en México", Documento de Investigación, núm. 9605, Banco de México, México.

Greenhalgh, C. (1990), "Innovation and Trade Performance in the United Kingdom", The Economic Journal, Vol. 100, Londres, pp. 105-118.

Grossman, G., y E. Helpman (1995), “Technology and trade”, en Jones, R., y P. Kenen (comps.), op. cit., primera edición, Vol. 3, pp. 1279-1337. 
Grossman, G., y Q. Li (2009), “Exporting, R\&D, and Absorptive Capacity in UK Establishments", Oxford Economic Papers, Vol. 61, Oxford, pp. 74-103.

Harris, R., y Q. Li (2009), "Exporting R\&D, and Absorptive Capacity in UK Establishments", Oxford Economic Papers, Vols. 61-1, pp. 74-103.

Helpman, E. (1981), "International Trade in the Presence of Product Differentiation, Economies of Scale and Monopolistic Competition", Journal of International Economics, Vol. 11-3, Madison, Wisconsin, pp. 305-340.

— y P. Krugman (1985), "Market Structure and Foreign Trade: Increasing Returns, Imperfect Competition and International Economy”, MIT Press, Cambridge, p. 271.

Johansen, S., y K. Juselius (1990), "Maximum Likelihood Estimation and Inference on Cointegration with Applications to the Demand for Money", Oxford Bulletin of Economics and Statistics, Vol. 52, núm. 2, Oxford, pp. 169-210.

Krugman, P. (1980), "Scale Economies, Product Differentiation and the Pattern of Trade”, American Economic Review, Vol. 70, Pittsburgh, Pennsylvania, páginas 950-959.

_ (1983), "New Theories of Trade Among Industrial Countries", American Economic Review, Papers and Proceedings, Núm. 73, Pittsburgh, Pennsylvania, pp. 343-347.

Lall, S. (2000), “The Technological Structure and Performance of Developing Country Exports, 1985-1998”, Oxford Development Studies, Vol. 28-3, Oxford University, Oxford, pp. 337-369.

Lefebvre, E., M. Bourgault, y L. Lefebvre (1998), “R\&D Related Capabilities as Determinants of Export Performance", Journal of Small Business Economics, Vol. 10, Amsterdam, 365-377.

Love, J., y M. Mansury (2009), "Exporting and Productivity in Business Services: Evidence from the United States", International Business Review, Vol. 18-2, Londres, pp. 630-642.

Madsen, J. (2004), “Technological Revolutions, Innovations and Trade Performance", EPRU Working Paper Series, Núm. 2004-12, University of Copenhagen, Dinamarca.

- (2007), "Innovations and Manufacturing Export Performance in the OECD Countries", Oxford Economic Papers, Vol. 60, Oxford, pp. 143-167.

Magnier, A., y J. Toujas-Bernate (1994), “Technology and Trade: Empirical Evidences for the Major Five Industrialized Countries", Review of World Economics, 130, Kiel, Alemania, pp. 494-520.

Montobbio, F., y F. Rampa (2005), “The Impact of Technology and Structural Change on Export Performance in Nine Developing Countries", World Development, Vols. 33-34, Montreal, pp. 527-547. 
Narula, R., y K. Wakelin (1998), "Technological Competitiveness, Trade and Foreign Direct Investment”, Structural Change and Economic Dynamics, Vol. 9-3, Maryland, pp. 373-387.

OCDE (2007), "Moving-Up the Value Chain: Staying Competitive in the Global Economy", París.

Papanastassiou, M., y R. Pearce (1990), "Host Country Characteristics and the Sourcing Behaviour of the UK Manufacturing Industry", Discussion Papers in International Investment and Business Studies, Vol. 2-140 (mimeo), Reading, Reino Unido.

Romijn, H. (1997), “Acquisition of technological capability: A quantitative case-study of Pakistan's capital goods sector", World Development, Vol. 25-3, Montreal, pp. 359-377.

Roper, S., y J. Love (2002), "Innovation and export performance: evidence from UK and German manufacturing plants", Research Policy, Vol. 31-7, Londres, pp. 10871102.

Soete, L. (1987), "The impact of Technological Innovation on International Trade Patterns: The Evidence Reconsidered", Research Policy, Vol. 16-2, Londres, pp. 101-130.

Sousa, C., F. Martínez, y F. Coelho (2008), "The Determinants of Export Performance: A Review of the Research in the Literature Between 1998 and 2005", International Journal of Management Reviews, Vol. 10-4, Londres, pp. 343-374.

UNCTAD (2004), "Export Performance and its Determinants: Supply and Demand Constraints", Policy Issues in International Trade and Commodities, Study Series núm. 26, Ginebra.

Van Dijk, M. (2002), "The Determinants of Export Performance in Developing Countries: The Case of Indonesian Manufacturing”, Eindhoven Centre for Innovation Studies, Working Paper, núm. 02-01, Eindhoven, Países Bajos.

Van Hulst, N., R. Mulder y L. Soete (1991), "Exports and Technology in Manufacturing Industry", Review of World Economics, Vol. 127-2, Kiel, pp. 246-264.

Verspagen, B., y K. Wakelin, (1997), "International Competitiveness and its Determinants", International Journal of Applied Economics, vol. 11-2, Louisiana, EUA, pp. 177-190.

Wakelin, K. (1998), "Innovation and Export Behavior at the Firm Level”, Research Poli$c y$, Vol. 26, Londres, pp. 829-841. 